\title{
Group-level inference of information-based measures for the analyses of cognitive brain networks from neurophysiological data
}

Etienne Combrisson', Michele Allegra', Ruggero Basanisi', Robin A. A. Ince², Bruno Giordano', Julien Bastin ${ }^{3}$, Andrea Brovelli ${ }^{1}$

1 Institut de Neurosciences de la Timone, Aix Marseille Université, UMR 7289 CNRS, 13005, Marseille, France

2 Institute of Neuroscience and Psychology, University of Glasgow, Glasgow, UK

3 Univ. Grenoble Alpes, Inserm, U1216, Grenoble Institut Neurosciences, 38000 Grenoble, France

Corresponding authors:

Etienne Combrisson

e.combrisson@gmail.com

Institut de Neurosciences de la Timone (INT),

UMR 7289 CNRS, Aix Marseille University,

Campus de Santé Timone,

27 Bd. Jean Moulin,

13385 Marseille, France

Andrea Brovelli

andrea.brovelli@univ-amu.fr

Institut de Neurosciences de la Timone (INT),

UMR 7289 CNRS, Aix Marseille University,

Campus de Santé Timone,

27 Bd. Jean Moulin,

13385 Marseille, France 


\section{Manuscript details}

Number of pages: 47

Number of figures: 9

Number of tables: 0

Number of words: 263 (abstract), 827 (introduction), 2726 (discussion)

\section{Abbreviations}

FFX: Fixed Effect

RFX: Random Effect

MC : Multiple Comparisons

IT : Information-theory

MI : Mutual-information

ML : Machine-learning

MEG : Magnetoencephalography

sEEG : stereoelectroencephalography

FC: Functional Connectivity

DFC: Dynamic Functional Connectivity

GC: Granger causality

BIDS : Brain Imaging Data Structure

\section{Keywords}

group-level statistics; information-based measures; cluster-based; non-parametric; python; reproducibility; neurophysiology

\section{Author contributions}

Conceptualization : EC, BG, RAAI, AB

Data curation : EC, JB

Formal analysis : EC

Funding acquisition : $A B$

Investigation : $E C, J B, A B$

Methodology : EC, BG, RAAI, MA, AB

Project administration : $E C, A B$

Resources: $A B$

Software: EC, RB, RAAI, AB

Supervision : $A B, B G, R A A I$

Validation :

Visualization : EC

Roles/Writing - original draft : EC, BG, AB

Writing - review \& editing : $M A, R A A I, A B$ 
bioRxiv preprint doi: https://doi.org/10.1101/2021.08.14.456339; this version posted August 15, 2021. The copyright holder for this preprint (which was not certified by peer review) is the author/funder, who has granted bioRxiv a license to display the preprint in perpetuity. It is made available under aCC-BY-NC-ND 4.0 International license. 
bioRxiv preprint doi: https://doi.org/10.1101/2021.08.14.456339; this version posted August 15, 2021. The copyright holder for this preprint (which was not certified by peer review) is the author/funder, who has granted bioRxiv a license to display the preprint in perpetuity. It is made available under aCC-BY-NC-ND 4.0 International license.

\section{Graphical abstract}

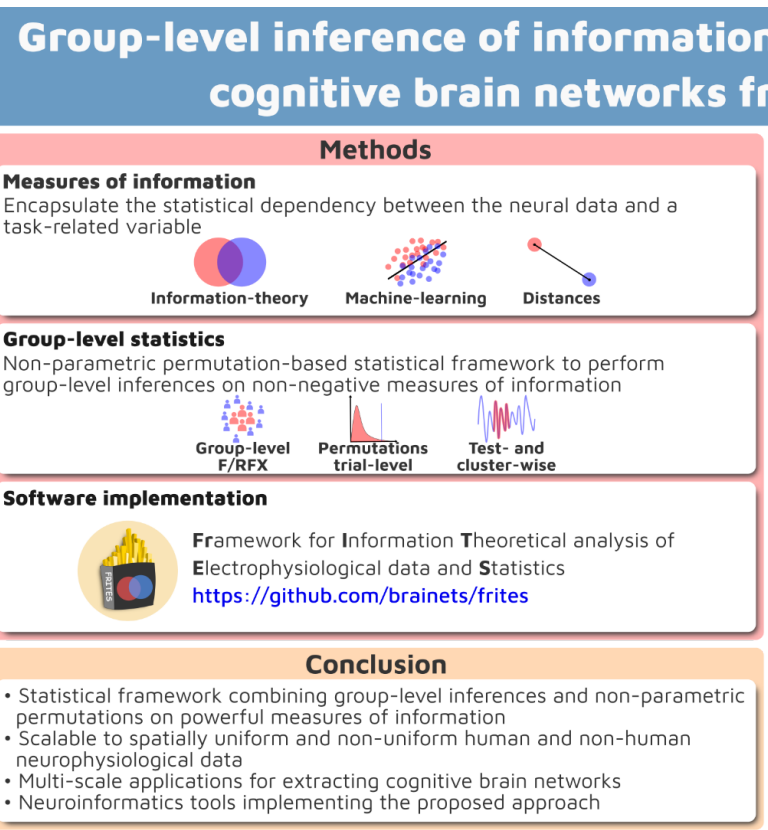

\section{cognitive brain networks f \\ Machine-learn \\ Distances}

\section{Measures of information}

Encapsulate the statistical dependency between the neural data and a

Information-theory

\section{Group-level statistics}

Non-parametric permutation-based statistical framework to perform

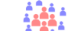

Group-level
F/RFX

Test- and
cluster-wise

\section{Software implementation}

Electrophysiological data and Statistics

https://github.com/brainets/frites

\section{Conclusion}

permutations on powerful measures of information

neurophysiological data

- Neuroinformatics tools implementing the proposed approach 


\section{Highlights}

- We introduce a statistical framework for the characterisation of cognitive brain networks through group-level inference of measures of information computed on neurophysiological data

- The framework supports both fixed- and random-effect models, test- and cluster-wise corrections based on non-parametric permutations

- The framework can serve statistical inferences at multiple levels from local brain regions to inter-areal functional connectivity and graph theoretical metrics

- A Python open-source toolbox called Frites includes the proposed statistical methods (https://github.com/brainets/frites) 


\section{Abstract}

The reproducibility crisis in neuroimaging and in particular in the case of underpowered studies has introduced doubts on our ability to reproduce, replicate and generalize findings. As a response, we have seen the emergence of suggested guidelines and principles for neuroscientists known as Good Scientific Practice for conducting more reliable research. Still, every study remains almost unique in its combination of analytical and statistical approaches. While it is understandable considering the diversity of designs and brain data recording, it also represents a striking point against reproducibility. Here, we propose a non-parametric permutation-based statistical framework, primarily designed for neurophysiological data, in order to perform group-level inferences on non-negative measures of information encompassing metrics from information-theory, machine-learning or measures of distances. The framework supports both fixed-and random-effect models to adapt to inter-individuals and inter-sessions variability. Using numerical simulations, we compared the accuity in ground-truth retrieving of both group models, such as test- and cluster-wise corrections for multiple comparisons. We then reproduced and extended existing results using both spatially uniform MEG and non-uniform intracranial neurophysiological data. We showed how the framework can be used to extract stereotypical task- and behavior-related effects across the population covering scales from the local level of brain regions, inter-areal functional connectivity to measures summarizing network properties. We also present a open-source Python toolbox called Frites ${ }^{1}$ that includes all of the methods used here, from functional connectivity estimations to the extraction of cognitive brain networks. Taken together, we believe that this framework deserves careful attention as its robustness and flexibility could be the starting point toward the uniformization of statistical approaches.

\footnotetext{
${ }^{1}$ https://aithub.com/brainets/frites
} 


\section{1) Introduction}

Modern theories suggest that cognitive functions emerge from the dynamic coordination of neural activity over large-scale and hierarchical networks (Bressler and Menon, 2010; Reid, 2019; Varela et al., 2001; von der Malsburg et al., 2010). A standard approach in cognitive brain networks analysis involves the characterisation of brain regions and inter-areal interactions that participate in the cognitive process under investigation (Battaglia and Brovelli, 2020; Bijsterbosch et al., 2020). Measuring the emergence of cognitive brain networks therefore underlies linking brain data to experimental variables, such as sensory stimuli or behavioral responses. Information-based measures, based on either information theory (Ince et al., 2017; Panzeri et al., 2008; Timme and Lapish, 2018) or machine learning (Glaser et al., 2019), provide ideal tools for quantifying the link between brain regions or network-level activity and task variables (Allefeld et al., 2016; Kriegeskorte et al., 2006; Kriegeskorte and Bandettini, 2007; Panzeri et al., 2017).

The neuroimaging community has relatively standardized statistical pipelines to identify task-related functional activations at the population level. Some examples are established softwares like SPM (Friston et al., 1994) and analysis pipelines developed in international initiatives, such as the Human Connectome Project. On the contrary, there exists a larger heterogeneity in statistical group-level approaches for the analysis of task-related neural representations from neurophysiological data. Such diversity mainly depends on a richer panel of modalities that can be used to record neurophysiological activity, each having unique spatiotemporal properties (e.g., EEG, MEG and intracranial EGG) and on differences in analysis goals (e.g., single-area versus network-level analyses). As an example, invasive intracranial recordings, which provide an exceptional signal-to-noise ratio at the single-trial level, are characterised by a spatially non-uniform sampling due to unique electrode implantations, which complexifies inferences at the population level and network level. On the other 
hand, M/EEG data provides an excellent technique for the analysis at the whole-brain level with the additional benefit of having an equal spatial sampling.

The reproducibility crisis in neuroimaging has many potential causes including analytic variability, statistical power and test-retest reliability (Poldrack et al., 2020). Taken together, these issues are limiting the ability of research groups to reproduce, replicate and generalize robust results (Arnold et al., 2019; Niso et al., this issue). Concerning neurophysiological M/EEG and intracranial data, several solutions have been proposed, from a uniformization of brain data organization through BIDS formatting (Holdgraf et al., 2019; Niso et al., 2018; Pernet et al., 2019) to overall good scientific practices and recommendations for data sharing, analysis and reporting results (Pernet et al., 2020, 2018). The problem of underpowered studies has been highlighted as one of the core limitation explaining the lack of reproducible results (Button et al., 2013; loannidis et al., 2014; Poldrack et al., 2017; Szucs and loannidis, 2017). In response, collaborative replication efforts like \#EEGManyLabs (Pavlov et al., 2021) are attempting to replicate the findings of a subset of the most influential EEG studies using larger cohorts to improve confidence in most cited results. While such large-scale projects combined to data-sharing are currently tackling the low statistical power problem, the sparsity of statistical group-level approaches and multiple hypothesis testing have also been highlighted as core limitations for reproducibility (Carp, 2012; Gilmore et al., 2017; Puoliväli et al., 2020). Therefore, there is a need for unifying statistical framework for extracting task-related neural representations both for single-area and network systems (Bassett et al., 2020) using information-based measures combined with a statistical layer accounting for the heterogeneity in the population (Mumford and Nichols, 2006) with the ability to adapt to the diversity of neurophysiological recordings.

Here, we address these issues by extending an existing statistical framework for group-level inferences on information-based measures (Giordano et al., 2017) to encompass neurophysiological recordings with both homogeneous spatial samplings across participants (or sessions), such as whole-brain source 
level data M/EEG data and spatially sparse recordings, such as intracranial sEEG, ECoG and LFPs. This framework supports any type of measure of information and can be used at the level of single brain area activity, connectivity-level inter-areal interactions (e.g., (un)directed functional connectivity measures) and network-level (e.g., on graph-theoretical measures). We tested the workflow on simulated data to compare fixed-versus random-effect group inference with testor cluster-wise p-values correction. We then re-analyzed cortical high-gamma activity (HGA) data from different recording modalities: a MEG experiment (Brovelli et al., 2015), where we successfully retrieved the visuomotor network using information metrics from both IT and ML and an intracranial experiment (Gueguen et al., 2021), revealing the outcome-related activity of the anterior insula during probabilistic learning. Finally and as a contribution toward reproducibility, we also provide to the community the neuroinformatics tools wrapped in an open-source Python toolbox called Frites ${ }^{2}$ (Framework of Information Theoretical analysis of Electrophysiological data and Statistics). This package includes the proposed group-level statistical framework such as functions for the estimation of functional connectivity. By default, Frites is using metrics from the IT but it can be extended to any type of measure of information.

\footnotetext{
${ }^{2}$ https://brainets.github.io/frites/
} 


\section{2) Methods}

\subsection{Statistical framework for group-level inferences of information-based measures}

The main objective of the current work was to develop a non-parametric framework to assess the group-level statistical significance on measures of information computed on neural correlates of individual brain areas or interareal relations, such as functional connectivity (FC) measures. The proposed framework (Figure 1) relies on two main steps: (i) the estimation of the true and permuted effect size using a measure of information; (ii) statistical inference at the test-wise (i.e., at a single time-point) or cluster-level (Maris and Oostenveld, 2007).

\subsubsection{Effect size estimation and permutation tests for fixed and random effect models}

The estimation of effect size on a population depends on assumptions about the underlying distribution and inter-subject variability. A fixed-effect (FFX) is a single-level model assuming that each subject brings the same fixed contribution to the observed effect (Friston et al., 1999). It extracts the typical characteristics of a sample, which explain why it is massively used for reporting case studies. A FFX at the population level can be estimated by computing the effect size across all concatenated subjects and trials (Penny and Holmes, 2007). This is equivalent to considering the data as coming from a single subject, and discards random variations across subjects. While FFX shines by its simplicity, conclusions that can be drawn only concern the subjects that constitute the available sample as a whole, and therefore can not be generalized to the population-level. On the contrary, a random-effect (RFX) is a hierarchical model consisting in : (i) extraction of single-subject effect size and (ii) fitting a model at the population level. Here, the sample of subjects is considered as a subsample of a broader population and then, if new subjects are added, their effect size should fall into the estimated model (Figure 1). 
In our approach, significance for fixed- or random-effects was performed using a nonparametric approach based on permutations. Permutation-based significance testing shortcuts distributional assumptions by relying on the exchangeability hypothesis (Nichols and Holmes, 2002). Since the aim is to quantify task-related effects, permutations are performed by randomly swapping the task-related variable, as usually performed in other fields (Combrisson and Jerbi, 2015). For FFX analyses, the estimation of the null distribution of effect sizes assumes exchangeability of results from different participants and trials, and it is implemented by means of random permutation of data across both. For RFX analyses, we assume exchangeability of results from different trials only, and permute trial-specific results independently for each participant. We test the population mean with a non-parametric test using the t-statistic. We obtain a non-parametric null distribution for the population t-statistic by sampling a trial-permuted value from each participant. As information-based metrics are often subject to bias, it is not correct to perform a parametric population level t-test against a specific value (such as 0 ). Obtaining a non-parametric population null distribution from within-participant trial permutations avoids this problem, as the permutation values are obtained from the same number of samples, and so will be subject to the same level of bias. FFX and RFX effects corresponding to the true effect size and the distributions of permutations corresponding to the effect size that can be obtained by chance. However, for the FFX, the summary statistics at the population level is expressed in the unit of the information-based method (i.e in bits when using IT metrics or, for example, in percentage for ML decoding) while for the RFX it corresponds to T-values (Figure 1).

\subsubsection{Inferring p-values and correcting for multiple comparisons}

In order to assess whether the estimated effect size significantly differs from the chance distribution and to correct for multiple comparisons (Cao and Zhang, 2014; Lindquist and Mejia, 2015; Nichols and Hayasaka, 2003), we implemented both test- and cluster-wise statistics (Maris and Oostenveld, 2007). In the case of test-wise correction, $p$-values are defined as the proportion of surrogates that are exceeding the observed true effect size estimation. The 
p-values are then corrected for multiple comparisons using the False Discovery Rate (Genovese et al., 2002) or the maximum statistics, which provides a threshold that controls family-wise error rate (Holmes et al., 1996; Nichols and Holmes, 2002). For cluster-level statistics, the cluster forming threshold is defined as the 95th percentile across all of the permutations. Such threshold is used to identify the clusters on both the true and permuted data. In order to infer the $p$-values and to correct for multiple comparisons, a null distribution composed of the maximum of the cluster mass is built and the corrected $p$-values are obtained by assessing the proportion of cluster masses computed on the surrogates that exceed the cluster mass of the true effect size.

For simplicity, the description above about the statistical framework is considering the participant as the fixed or random variables. The same description still holds if sessions are used instead or, in the context of intracranial data, the recording contacts across participants (Gueguen et al., 2021). 


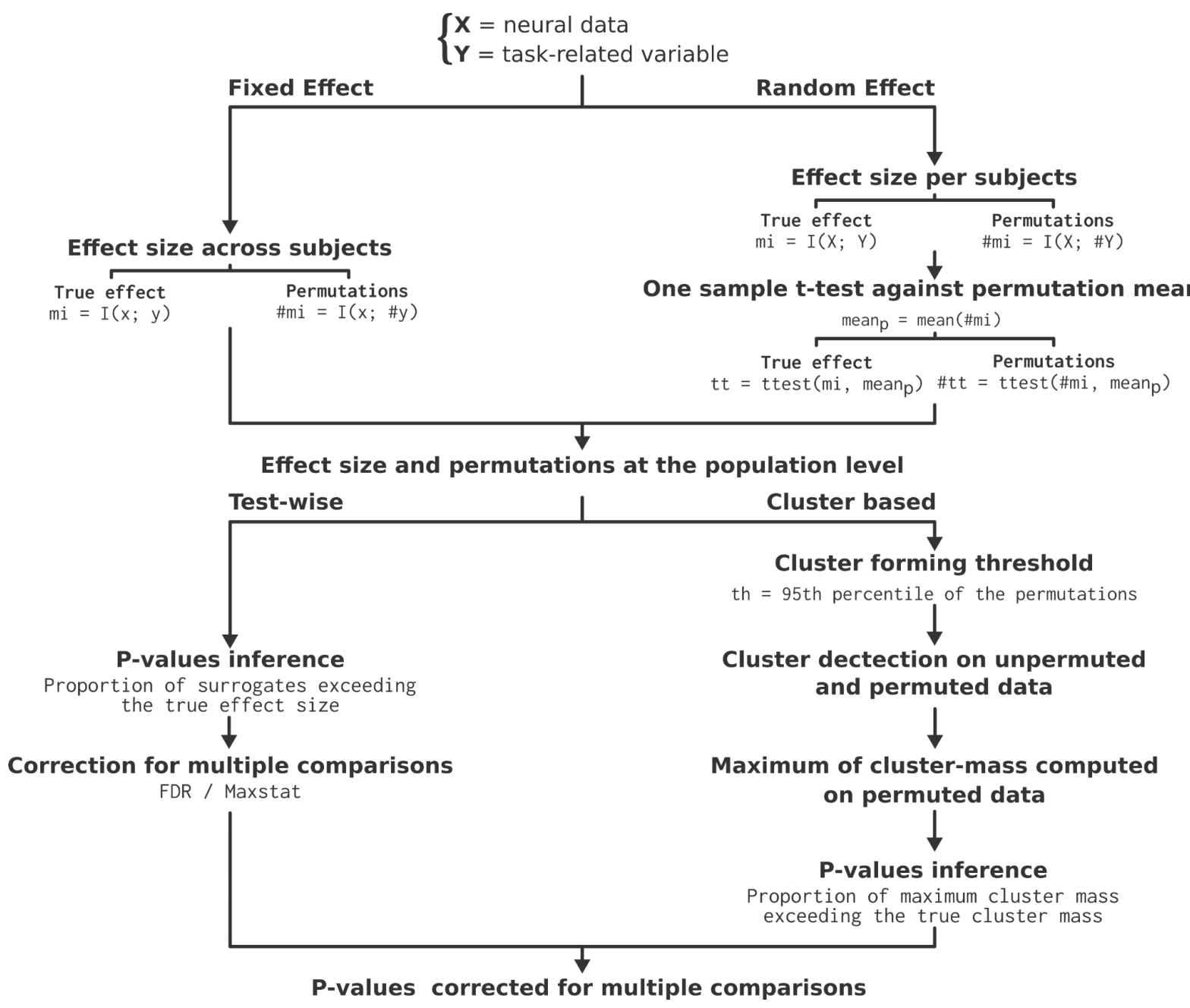

Figure 1. Statistical framework for group-level inferences using information-theoretic measures (I=Mutual-Information) to extract task-related neural activity or functional connectivity. The upper branches describe the fixed and random effect implementations, both leading to a measure of a true effect size and the estimation of the null distribution. Then, starting from the effect size and the permutations, bottom branches describe how both are used to infer and the p-values corrected for multiple comparisons using either test- or cluster-wise corrections.

\subsection{Measuring information for the analysis of cognitive brain networks}

Information theory (Cover and Thomas, 1991) represents one of the most successful and promising data-driven frameworks to quantify task-related information in neural signals (Borst and Theunissen, 1999; Quiroga and Panzeri, 2009) and it has been extensively applied to neurophysiological signals (Cogan et al., 2011; Giordano et al., 2017; Gross et al., 2013; Ince et al., 2016; Jeong et al., 2001; Magri et al., 2009; Schyns et al., 2011) and neuroimaging studies (Ince et al., 2017; Ostwald and Bagshaw, 2011; Panzeri et al., 2008; Timme and Lapish, 2018; Wibral et al., 2015). In addition, information theoretic measures based on 
the Wiener-Granger principle (Bressler and Seth, 2011; Brovelli et al., 2004; Ding et al., 2006; Seth et al., 2015), such as Transfer Entropy (Schreiber, 2000) and Directed Information (Massey, 1990), provide means to quantify network-level directional influences and can capture arbitrary (linear and nonlinear) time-lagged conditional dependencies between neural signals (Besserve et al., 2015; Ince et al., 2015; Vicente et al., 2011). Although our statistical approach can be applied to all information-based metrics, we focused our analysis and application to information theoretical quantities, such as mutual information (MI) and conditional MI (CMI) measures (Ince et al., 2012).

The standard method to compute entropies for the calculation of mutual information (MI) and conditional MI (CMI) is the binning method. Such "direct" method consists in binning each univariate variable and collecting the bin counts, so to approximate the joint probability distribution $P(X, Y)$ by the multidimensional histogram (Beirlant et al., 1997; Treves and Panzeri, 1995). However, binning strongly depends on the number of samples and suffers from the curse of dimensionality (Geman et al., 1992). Binless strategies have also been proposed, such as Kernel Density Estimation (KDE) methods (Moon et al., 1995). However, KDE estimations suffer the same limitations of direct methods linked to the curse of dimensionality typical of high-dimensional datasets, such as neurophysiological data. A promising alternative based on semi-parametric estimation techniques has been recently proposed, namely the Gaussian Copula Mutual Information (GCMI) (Ince et al., 2017). In short, Ml does not depend on the marginal distributions of the variables, but only on the copula function which describes their dependence. GCMI therefore first requires transforming the inputs so that the marginal distributions are a standard normal. This copula-normalisation step involves calculating the inverse standard normal cumulative density function (CDF) value of the empirical CDF value of each sample, separately for each input dimension, before using a parametric, bias-corrected, Gaussian Ml estimator (i.e., sum-rank computation). This provides a lower bound estimate of the Ml, without making any assumption on the marginal distributions of the input variables. It is important to stress that the rank-based copula-normalisation preserves the relationship between variables as long as this relation is monotonic (i.e., strictly increasing or 
decreasing). For example, if the $Y$ variable is a parabolic function of $X$, the ranking is not preserved, and thus the relation between them. In other words, for 1D variables the GCMI is capable of detecting linear and non-linear relations, as long as the relation is monotonic.

Nevertheless, GCMI has several advantages for brain network analysis. The first advantage is the simplicity of the computation, which renders the algorithms applicable to large datasets with hundreds of variables (e.g., brain regions) and suitable once combined to non-parametric permutations. The second advantage is the ability to estimate entropies on few data samples. This property allows the estimation of $\mathrm{Ml}$ and $\mathrm{CMl}$ from short time series containing hundreds of time points, e.g., single trials or across trials. A third advantage is the possibility to compute $\mathrm{MI}$ and CMI between a combination of discrete and continuous variables, that can either be univariate or multivariate, with resulting effect sizes on a common and interpretable scale. This provides a tight link between information theoretic quantities and classical statistical approaches used in neuroscience. For example, if both variables are continuous and univariate (e.g., two time-series, one in the parietal and one in the occipital), $\mathrm{Ml}$ is related to the generalized Pearson correlation. If $X$ is a continuous variable (e.g., the high-gamma activity in the visual cortex) and $Y$ is discrete and univariate (e.g., task-related variable), their $\mathrm{MI}$ is equivalent to a Student t-test and it is related to a decoding machine-learning analysis where $X$ is the array of samples and $Y$ the vector of labels. Furthermore, the CMI produces an equivalent of partial correlation. For a more detailed description of the relation between information theoretic quantities and other statistical approaches, see the Table I in Ince et al. 2017.

\subsection{Software implementation}

We implemented the framework presented in this paper in an open-source python package called Frites ${ }^{3}$ (Framework for Information Theoretical analysis of Electrophysiological data and Statistics). The key objective of Frites is to identify task-related neural activity or cognitive brain networks through information

\footnotetext{
${ }^{3}$ https://brainets.qithub.io/frites/
} 
theory and also to be able to draw inferences at the group level. Frites contains two important classes. The first one is the workflow of Ml (frites.workflow. WfMi ${ }^{4}$ ) to quantify the statistical dependency between the neural data and a task-related variable. This workflow estimates the effect-size and the surrogates as shown in the top part of Figure 1. By default and for efficient computations, Frites is using a tensor-based implementation of the GCMI. However, custom estimators can be defined and provided to estimate effect sizes with for example decoders, measures of distance, correlations etc. Then, the effect-size and the surrogates are plugged into the second important workflow for group-level statistical inferences (frites.workflow.WfStats ${ }^{5}$ ), which corresponds to the bottom part of

Figure 1. Cluster-based correction is performed using the open-source software MNE-Python (Gramfort et al., 2013). One important aspect is that, in contrast to fMRI data, electrophysiological recordings do not necessarily have a spatial contiguity (e.g sparse intracranial data). Therefore, when using cluster-based corrections, the cluster forming threshold is inferred across time and space but then, the clusters are only detected across time. Finally, for correcting for multiple comparisons, the maximum of the cluster masses is taken across time and space.

\subsection{Numerical simulations}

We performed numerical simulations to generate artificial task-related data and then measure the performance of the group-level statistics when combined with information-based measures. The simulated data consisted in two variables: a multivariate variable $X$, which simulates the spatio-temporal structure of electrophysiological data and a univariate variable $Y$, reflecting a task-related continuous variable. At each time point, both $X t a n d Y t$ were defined as standard random Gaussian variables without temporal autocorrelation. Then, we introduced a statistical dependency between those two variables during a temporal interval $\left[t_{1} ; t_{2}\right]$ :

$$
X_{\left[t_{1} ; t_{2}\right]}=\operatorname{sign} \times Y+\sqrt{\frac{1-\operatorname{cov}^{2}}{\operatorname{cov}^{2}}} \times r
$$

${ }^{4}$ https://brainets.github.io/frites/api/generated/frites.workflow.WfMi.html

5 https://brainets.github.io/frites/api/generated/frites.workflow.WfStats.html 
where $r$ is a random gaussian noise of unit variance, sign that can either be -1 or 1 and cov a correlation parameter with values between ]0, 1] for controlling the correlation strength between $X$ and $Y$. If $\operatorname{cov}$ is 1 a perfect correlation is observed between $X$ and $Y$. On the contrary, the closer to zero cov is, the less the variables are correlated.

We defined three scenarios associated with different task-related effects whose amplitude and duration varied across time and space (Figure 2 ). For each scenario, a boolean ground-truth array was built with 0 and 1 indicating the absence or presence of effect, respectively, at a specific time and in a specific region. The objective was then to compare such a ground-truth array with the output statistics.

In addition to the above ground-truths describing various spatio-temporal profiles, we also measured the impact of four intertwined parameters of the simulated data, namely (i) the size of the population (i.e the number of subjects) (ii) the within-subject size (i.e the number of trials per subject (Baker et al., 2020)), (iii) the proportion of subjects having the effect and (iv) the inter-subject consistency or ISC. The ISC varied between 50 and 100\% and characterized the proportion of subjects with either positive $(\operatorname{sign}=1)$ or negative $(\operatorname{sign}=-1)$ correlation between the data and the stimulus. As an example, let us consider a dataset composed of 10 subjects, among which $80 \%$ has the effect ( 8 subjects out of 10). In such a case, an ISC of $50 \%$ means that 4 subjects have positive correlations, 4 have negative correlations and 2 have no effect. An ISC of 100\% means that, among the subjects having the effect, all of them have positive correlations.

In its most general definition, mutual information is capable of capturing any type of relation. However, Gaussian copula Ml between two continuous variables makes the assumption of a monotonic relation between the variables. When using FFX group-level approach, the $\mathrm{MI}$ is computed on data concatenated across subjects : this means that when positive and negative correlation coexist at the group level, the monotonicity assumption is broken. This does not occur in the 
RFX, as the latter computed MI independently for each subject. As a consequence, a low ISC (i.e with a balanced proportion of subjects having positive and negative correlations) should impact the FFX more than the RFX.

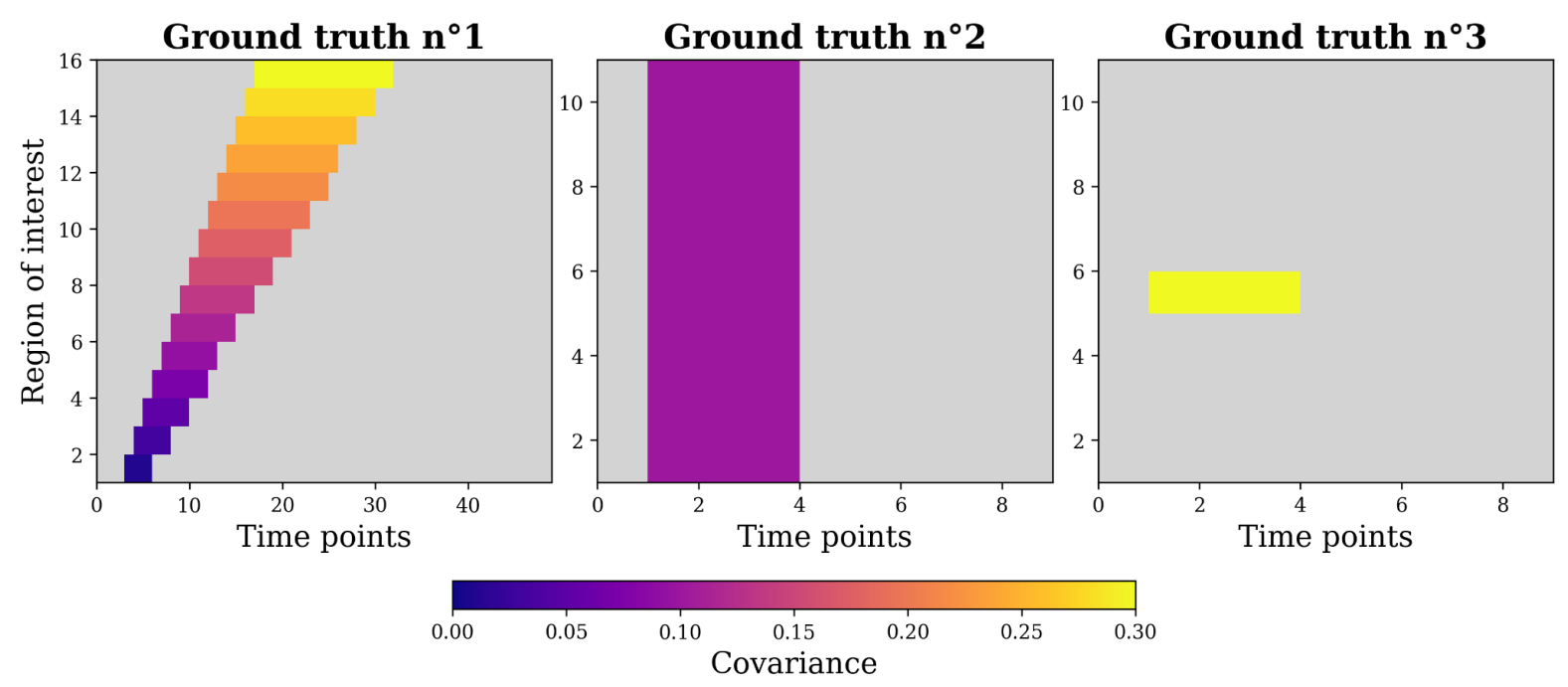

Figure 2. simulated spatio-temporal ground truths (GT) reflecting the presence and the strength of the statistical dependency between the data and the stimulus. The simulated effect is distributed across space (region of interest) and time points with a varying color-coded covariance. The higher the covariance, the easier the effect should be to be retrieved. The first GT simulates a spatially broad effect, with varying covariances and temporal cluster lengths. The second GT simulates a weak and diffuse effect and the third, a strong and focal effect. Gray parts symbolize the absence of effect.

The $\mathrm{p}$-values at the group-level for the $\mathrm{Ml}$ at each timepoint and brain region were inferred using the above-described permutation approach. We considered a significance threshold of $p<0.05$ to compute the fraction of true and false positives, and true and false negatives (TP, FP, TN, and FN, respectively). Those fractions were inferred by comparing the detected significant effects with the ground-truth. As an example, a TP results when measuring an Ml at $p<0.05$ corresponds to a simulated true effect. This procedure was repeated ten times in order to decrease the variability. We computed the sensitivity (or true positive rate) and specificity (or true negative rate) of the various approaches to group-level inference (Nichols and Hayasaka, 2003; Shapiro, 1999) defined as:

$$
\text { sensitivity }=\frac{T P}{T P+F N} ; \text { specificity }=\frac{T N}{T N+F P}
$$


In practice, sensitivity measures the overall accuracy for the detection of a true effect, and specificity measures the overall accuracy for the detection of the true absence of an effect. We then pooled information across measures of sensitivity and specificity to compute a summary measure of performance for the group-level method. To this purpose, we considered the Matthews Correlation Coefficient (MCC) (Matthews, 1975) defined as :

$$
M C C=\frac{T P \times T N-F P \times F N}{\sqrt{(T P+F P)(T P+F N)(T N+F P)(T N+F N)}}
$$

where MCC can assume any value in the $[-1,1]$ range, with 1 measuring a perfect performance, -1 measuring a perfect negative correlation and 0 a chance-level performance (Chicco and Jurman, 2020; Mensen and Khatami, 2013; Roels et al., 2016; Vihinen, 2012).

\subsection{Validation on MEG and intracranial sEEG electrophysiological data}

To validate the proposed statistical approach, we reanalysed human brain data previously published using standard parametric approaches. We analysed: i) source-level high-gamma activity (HGA) estimated from MEG data recorded during the execution of a visuomotor-mapping learning task (Brovelli et al., 2017, 2015) and (ii) HGA estimated from intracranial recordings (stereotactic electroencephalography, sEEG) acquired during a probabilistic learning task (Gueguen et al., 2021).

\subsubsection{MEG high-gamma activity during a visuomotor-mapping learning task}

We reanalysed HGA $(60-120 \mathrm{~Hz})$ estimated at the source level on ten right-handed healthy subjects performing a visuomotor-mapping task (Brovelli et al., 2015) consisting in executing finger movements instructed by a visual stimulus (digit 1 instructed the execution of the thumb, digit 2 for the index finger, digit 3 for the middle finger, and so on). Each participant performed two sessions of 60 trials each (total of 120 trials). MEG recordings were performed using a 248 magnetometers system (4D Neuroimaging, magnes 3600). Single-subject cortical 
parcellation was performed using the MarsAtlas brain scheme (Auzias et al., 2016). Single-trial and z-scored (with respect to a baseline period from -0.5 to $-0.1 \mathrm{~s}$ before stimulus onset) HGA time-series aligned on finger movement were estimated for each MarsAtlas' parcel, as described in previous studies (Brovelli et al., 2017). The goal of the original publication was to isolate the brain regions involved in the performance of arbitrary visuomotor associations. To this end, authors used linear mixed-effect models to contrast the HGA activity occurring during baseline against the HGA while performing the visuomotor association. The dynamic functional connectivity (DFC) was computed using the Pearson correlation over sliding windows of $500 \mathrm{~ms}$ stepped every $10 \mathrm{~ms}$.

In the present study, we investigated whether it was possible to retrieve the visuomotor-related cortical brain regions and network using only information-based measures combined with the proposed group-level statistical framework. To this end, we computed the MI between the single-trial HGAs for baseline (-0.6 to $-0.1 \mathrm{~s})$ and visuomotor-related periods. To highlight the generalizability of the proposed framework to other measures of information, we also used machine-learning (ML) outputs. For the $M L$ analysis, we used a Linear Discriminant Analysis classifier (LDA) with a stratified 10-fold cross-validation to split the data into training and testing sets. The LDA was used to decode whether the HGA was coming from the baseline or visuomotor-related period. A new classifier was systematically defined at each time point to produce time-resolved analysis. For summarizing the quality of predictions, we used the area under the curve (AUC) metric. ML analysis was performed using the Python software scikit-learn (Pedregosa et al., 2011).

For network-level analyses, we computed the single-trial DFC on HGA using the $\mathrm{Ml}$ over sliding windows of $500 \mathrm{~ms}$ stepped every $10 \mathrm{~ms}$ during both baseline and visuomotor-related periods. We then computed the $\mathrm{Ml}$ and $\mathrm{p}$-values between the DFC estimated during the $500 \mathrm{~ms}$ baseline and visuomotor-related periods. Finally, to illustrate the possibility of the proposed framework to analyse graph-theoretical metrics computed on DFC matrices, we investigated task-related differences of network measures by means of network efficiency, modularity and assortativity. Each measure was computed using the Brain 
Connectivity Toolbox (Rubinov and Sporns, 2010) on the binarized 2D matrix of FC, at the single-trial level and at each time point. Since it is not the core point of this paper, we binarized the matrix with an arbitrary threshold to keep 50\% remaining links. A more robust analysis would require investigating multiple thresholds (Rubinov and Sporns, 2010).

\subsubsection{Intracranial sEEG recordings during a probabilistic learning task}

We then investigated outcome-related effects in intracranial HGA, previously observed using general linear models (GLM). Previous analyses revealed differently modulated activity during the reward and punishment conditions in the anterior insula (aINS) (Gueguen et al., 2021). The dataset was composed of twenty patients with medically-intractable epilepsy, implanted with five to seventeen stereotactic electroencephalography (sEEG) electrodes. Patients performed a probabilistic instrumental learning task adapted from previous studies (Palminteri et al., 2012; Pessiglione et al., 2006). The task consisted in the presentation of visual stimuli leading either to monetary gains or losses. Subjects were instructed to maximize their financial payoff, while considering reward-seeking and punishment-avoidance strategies as equally important. During the task, four pairs of visual abstract cues taken from the Agathodaimon alphabet were presented on a screen. The four cue pairs were divided in two conditions ( 2 pairs of reward and 2 pairs of punishment cues), associated with different pairs of outcomes (winning $1 €$ versus nothing or losing $1 €$ versus nothing). Subjects performed three to six sessions during which each pair was presented 24 times, leading to a total number of trials across sessions between 288 and 576. SEEG data preprocessing was conducted according to routine procedures described in previous studies (Bastin et al., 2016; Combrisson et al., 2017; Jerbi et al., 2013, 2009). These included signal bipolarization, where each electrode site was re-referenced to its direct neighbor. Bipolar re-referencing can increase sensitivity and reduce artefacts by canceling out distant signals that are picked up by adjacent electrode contacts (e.g., mains power). Next, electrodes contaminated with pathological epileptic activity were systematically removed using visual inspection and time-frequency decompositions. Time-frequency 
analyses were carried out using MNE-Python software (Gramfort et al., 2013). Single-trials HGA aligned on outcome presentation were estimated using a DPSS tapers (central frequency of 100hz, 15 cycles, time bandwidth of 15 leading to a frequency range of $[50,150] \mathrm{Hz})$. A more detailed description of the data acquisition and the task design have already been described elsewhere (Gueguen et al., 2021). In the present study, we focused on the anterior insula and investigated whether the HGA was differently modulated during the reward and punishment conditions. 


\section{3) Results}

In the following sections, we will first present the results of numerical simulations assessing the performance of the workflows in statistically detecting various spatio-temporal profiles. We will then present the results obtained from the reanalysis of cortical HGA estimated from MEG data during visuomotor mapping and from intracranial data during reinforcement learning.

\subsection{Numerical simulations: accuracy comparison of the group-level approaches}

Three ground-truth scenarios (Figure 2) were simulated to compare the two group-level approaches (FFX and RFX) combined with one of the three methods for correcting p-values for multiple comparisons across time and brain regions, namely, the maximum statistics, the FDR or the cluster-based, respectively. We also varied the number of subjects and trials (Figure $\mathbf{3}$ ), such as the proportion of the subjects having the effect and the inter-subject consistency or ISC (Figure 4).

Figure 3 shows the Matthews Correlation Coefficient (MCC), an overall measure of performance (Sec. 2.4), as a function of the number of simulated subjects and trials. Overall, the FFX (Fig. 3A-C) leads to slightly higher values of MCC compared to the RFX (Fig. 3E-F) and across the three correction methods. This translates into a decrease in the data size requirements. For example, using the maximum-statistics at 200 trials, the FFX (Fig. 3A) reaches a MCC of 0.8 using approximately 12 subjects while the RFX (Fig. 3D) requires around 20 subjects. Additionally, for both the FFX and RFX, the cluster-based correction (Fig. 3C and F) performed systematically better than maximum statistics (Fig. 3A and D) and FDR (Fig. 3B and E). Note that we fixed the percentage of subjects having the effect at $80 \%$ and the ISC at $90 \%$. Therefore, this set of parameters simulates a scenario where the relation between the data and the stimulus is relatively uniform across subjects, which is in favour of the FFX's assumptions. 

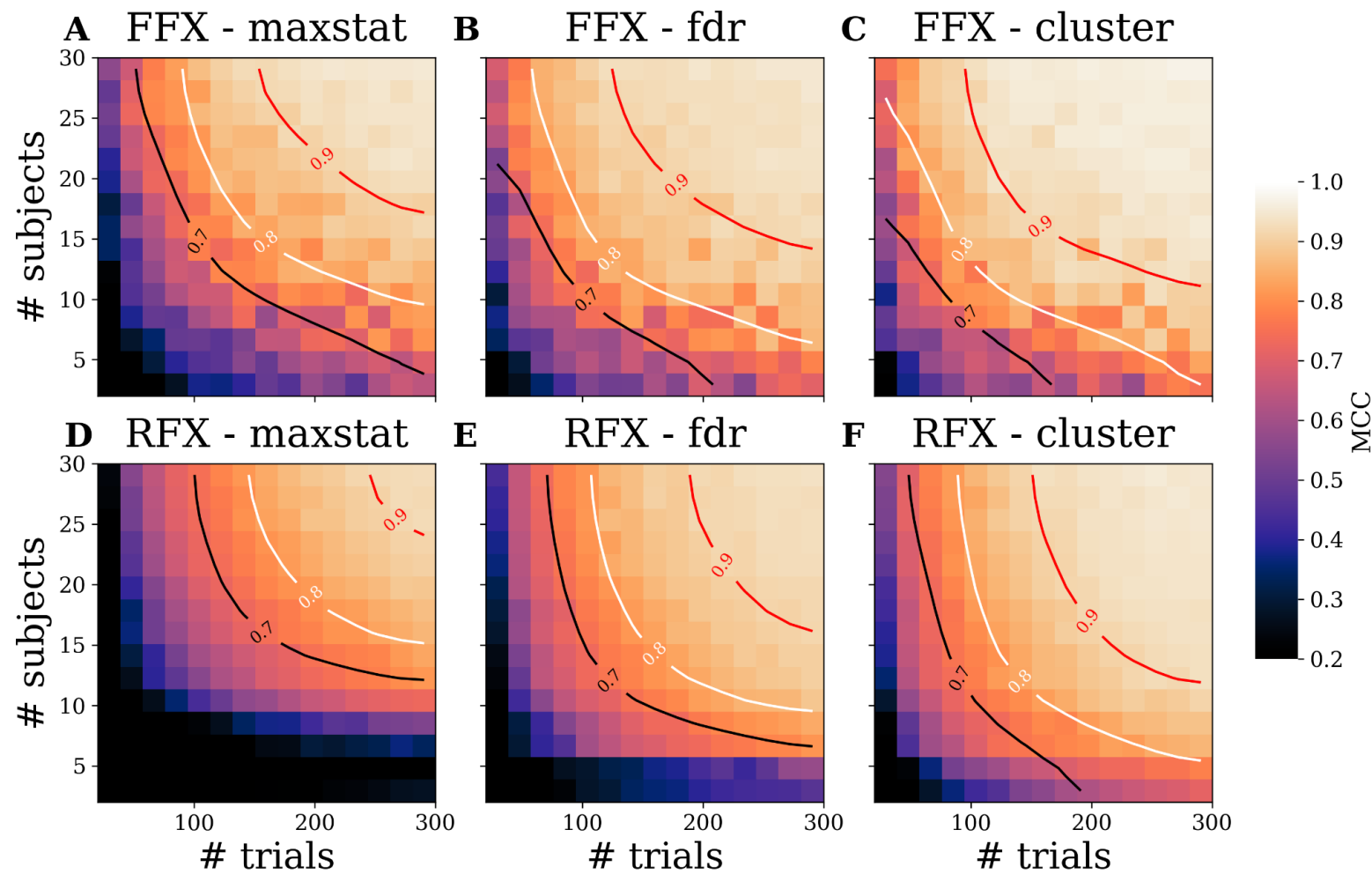

Figure 3. Comparison of group-level approaches on simulated data with varying number of trials (from 20 to 300) and number of subjects (from 2 to 30). The comparison includes the FFX $(A-C)$ and the RFX $(D-F)$, each combined with the three methods for correcting the p-values for multiple comparisons (maximum statistics, FDR and cluster-based) across time and brain regions. The MCC is computed across all three ground truths and the 10 repetitions, with $80 \%$ of the subjects having the effect and with a fixed ISC of $90 \%$. Red, orange and white lines respectively represent a MCC of $0.7,0.8$ and 0.9 .

We then investigated the dependence on an additional parameter relevant in group-level analyses, namely the heterogeneity across subjects, which was simulated by varying the inter-subject consistency (ISC). Figure 4 shows the MCC values as a function of the ISC (varying from 50 to 100\%) and the percentage of subjects having the effect that varies (varying from 10 to 100\%). A clear difference in dependence between the FFX and the RFX was observed as a function of the ISC. The MCC under the RFX model was stable for varying values of ISC (Fig. 4D-F). On the other hand, the FFX required that at least $80 \%$ of the subjects have the same type of effect in order to reach a MCC of 0.8 (i.e $80 \%$ with positive or negative correlations between the simulated data and the stimulus) (Fig. 4A-C). Nevertheless, for ISC values approximately higher than $90 \%$, the MCC 
scores were larger in the FFX than in the RFX setting. The second main difference concerned the correction for multiple comparisons. Fig. $\mathbf{4 C}$ and $\mathbf{F}$ show that cluster-based correction outperformed the maximum statistics and the FDR. Interestingly, the correction had a strong impact on the required proportion of subjects having the effect for the RFX (larger than $80 \%$ with the maximum statistics and $\sim 50 \%$ for the FDR and cluster-based).
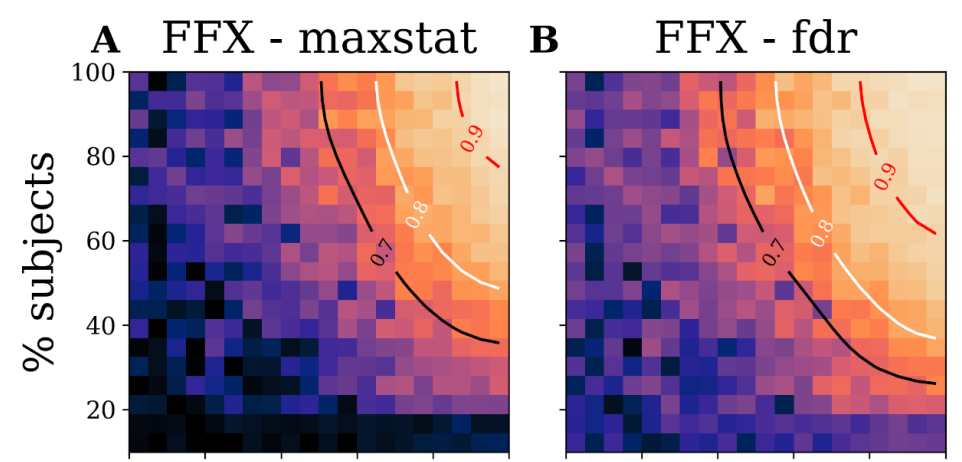

c FFX - cluster
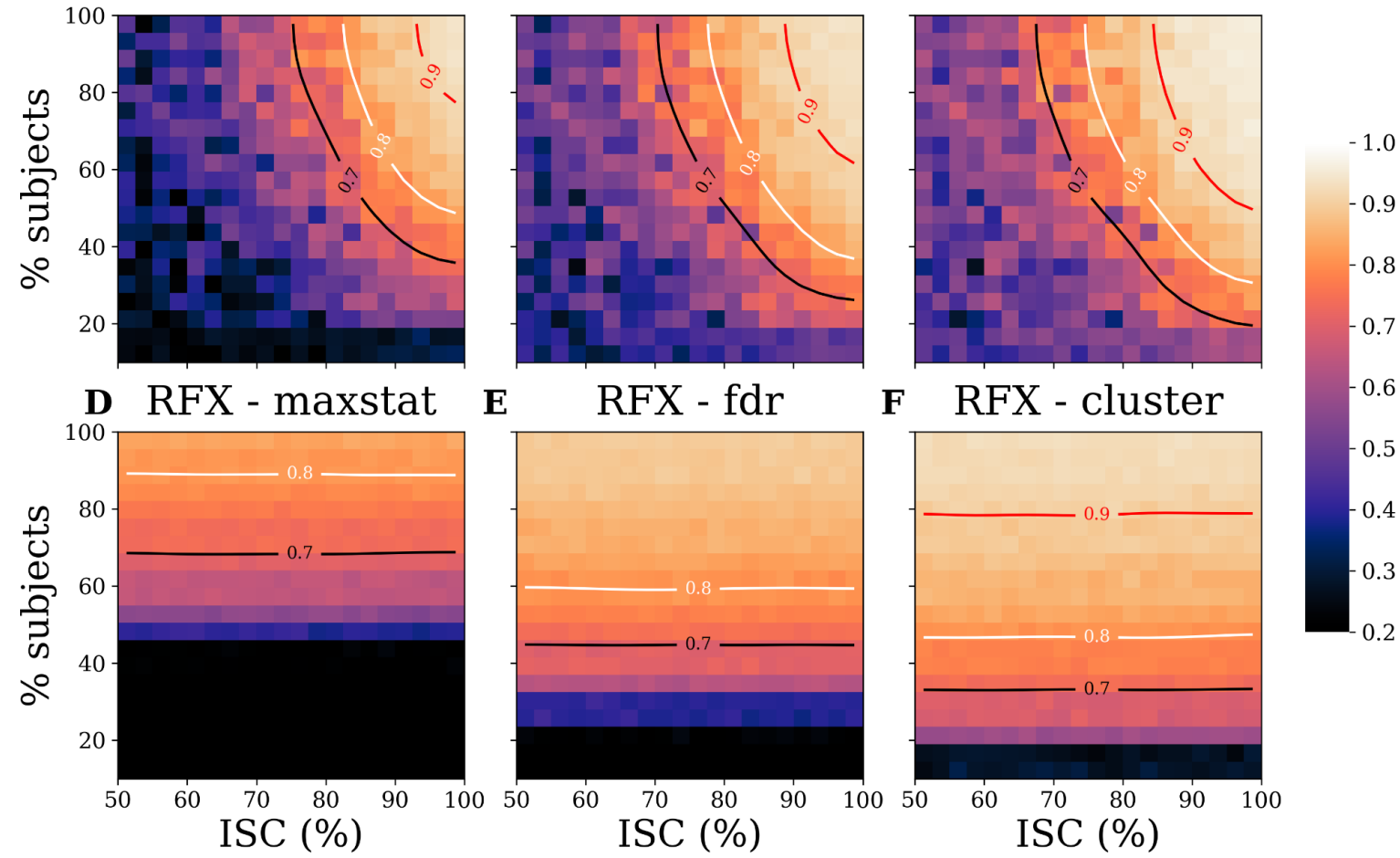

Figure 4. Comparison of group-level approaches on simulated data with varying inter-subject consistency (from 50 to 100\%) and proportion of subjects having the effect (from 10 to 100\%). The comparison includes the FFX $(A-C)$ and the RFX $(D-F)$, each combined with the three methods for correcting the $p$-values for multiple comparisons (maximum statistics, FDR and cluster-based) across time and brain regions. The MCC is computed across all three ground truths and the 10 repetitions, across 15 subjects and 200 trials. Red, orange and white lines respectively represent a MCC of $0.7,0.8$ and 0.9 .

\subsection{Brain data: extracting task-related cognitive brain networks using information-based measures from human neurophysiological data}

To validate the proposed statistical approach on human neurophysiological data, we reanalysed two previously-published datasets, which were analysed using standard parametric approaches. We analysed: (i) source-level high-gamma activity (HGA) estimated from MEG data recorded during the execution of 
visuomotor-mapping task (Brovelli et al., 2017, 2015) and (ii) high-gamma activity (HGA) computed from intracranial recordings (stereotactic electroencephalography, sEEG) acquired during a probabilistic learning task (Gueguen et al., 2021). For both modalities, we used the single-trial high-gamma activity (HGA) as the neural marker modulated by the task.

\subsubsection{Visuomotor-related brain network and MEG high-gamma activity}

We computed the MI between the single-trial HGAs and a discrete vector of conditions (baseline versus visuomotor-related periods). The aim was to identify which brain areas displayed visuomotor-related changes in HGA. We then performed a random-effect group-level analysis, where the mutual information was estimated for each subject and then a t-test was applied across subjects. The p-values were corrected for multiple comparisons using a cluster-based approach.

We reproduced the main findings of a previous study (Figure 2 in Brovelli et al., 2017) revealing a visuomotor-related large-scale network (Figure 5) involving bilateral dorsolateral and dorsomedial motor regions (Mdl, Mdm), premotor (PMdl, PMdm) and somatosensory areas (Sdl, Sdm). Parietal cortices also displayed differences in both hemispheres in the medial and superior parietal cortex (PCm, SPC, SPCm) such as in the mid, posterior and inferior portions of the cingulate cortex (MCC, PCC, ICC). Some activations were also significant only in the left hemisphere, namely the caudal dorsomedial part of the prefrontal cortex (PFcdm), the ventral somatosensory (Sv) such as the dorsal and ventral parts of the inferior parietal cortex (IPCd, IPCV). Finally, we found large differences in HGA within the occipital lobe, especially in the rostral medial and caudal medial part of the visual cortex (VCcm, VCrm) and cuneus (Cu) of both hemispheres. To a lesser degree, the superior and lateral parts of the visual cortex (VCs, VCl) also exhibited differences (Figure 5). It should be noticed that, around the movement onset, motor-related regions (Mdl, Mdm, PMdm, PMdl, Sdl) showed stronger differences between baseline and action in the left hemisphere compared to the right hemisphere, which is an expected result as the subject performed the task with the right hand. In the occipital lobe, highest values of $\mathrm{MI}$ were reached approximately $250 \mathrm{~ms}$ before movement onset. 


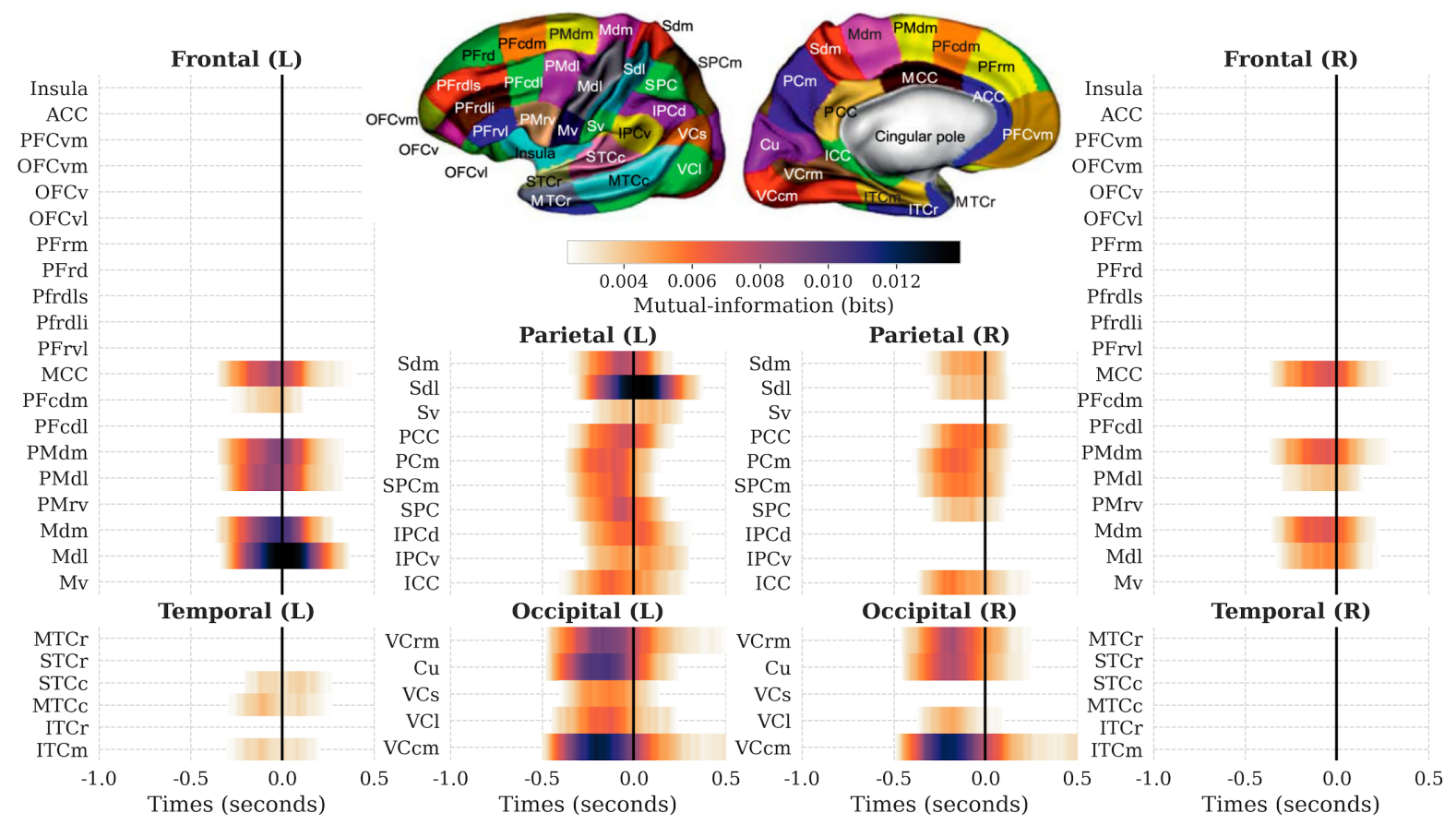

Figure 5. Brain areas showing significant differences of high-gamma activity between the action and the baseline. The plotted mutual-information is obtained by taking the mean across single-subject $\mathrm{Ml}$ estimations, at each time-point and parcel. P-values are corrected for multiple comparisons using a cluster-based correction and displayed temporal clusters are inferred by thresholding the $p$-values at a significant level of $p<0.05$. Data are aligned on movement onset represented here with a vertical black line at 0 seconds.

To illustrate the proposed group-level statistical method on non-information-theoretic measures, we applied it on machine-learning (ML) outputs where a new classifier was systematically redefined and retrained at each time point and brain region for each subject (Sec. 2.5.1). To this end, we performed the same analysis as above (classifying action versus baseline in somatosensory ( $\mathrm{Sdl}$ ), motor (Mdl) and visual (VCcm) parcels, all three located in the left hemisphere (Figure 6). Similarly to the Ml approach, permutations were computed by training and testing the classifier (LDA) on a randomly shuffled version of the label vector (Combrisson and Jerbi, 2015). Finally, we applied the random-effect group-level analysis on the AUC to infer p-values. Group-level statistics on both $\mathrm{Ml}$ and $\mathrm{ML}$ scores lead to very similar significant temporal clusters with the only exception of VCcm, where the significant cluster identified by $M L$ was narrower. 


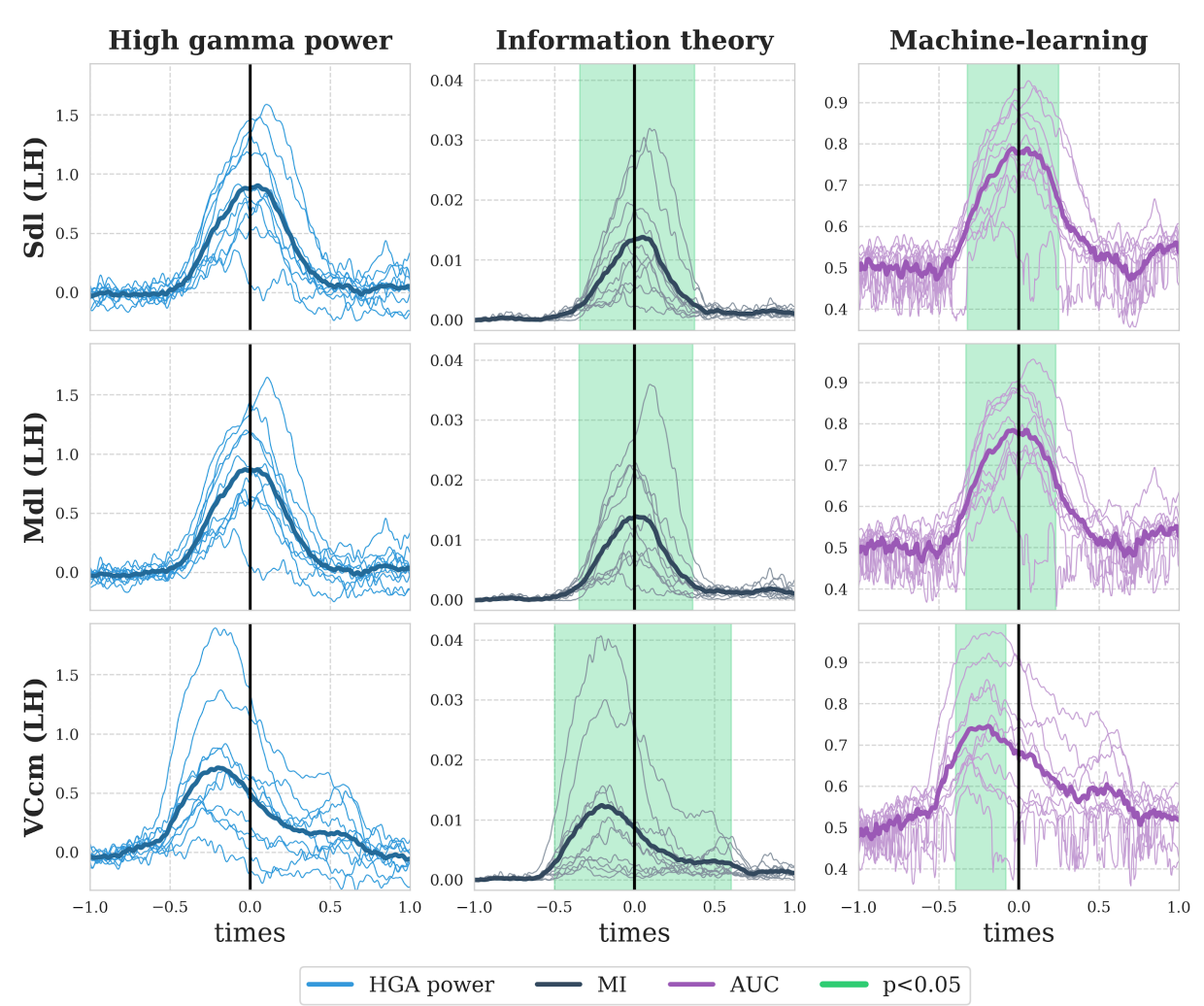

Figure 6. highlighting significant differences of high-gamma activity between the baseline and the action using group-level analysis on information theoretic and machine-learning metrics for three brain areas (dorsolateral somatosensory and motor cortex (Sdl, Mdl) and caudal medial visual cortex $(\mathrm{V} C \mathrm{~cm})$ ). The columns respectively refer to the high-gamma activity (blue), the mutual-information (black) and the machine-learning results (purple) expressed using the area under the curve (AUC). For all columns, thin lines stand for single-subject estimations and thick lines represent the mean across subjects. Green clusters highlight significant differences at $p<0.05$. Data are aligned on movement onset represented here with a vertical black line at 0 seconds.

We then tried to replicate previous visuomotor-related undirected and dynamic (time-resolved) functional connectivity (DFC) analyses (Figure 4 in Brovelli et al., 2017). We tested the hypothesis that interareal DFC was different during the execution of visuomotor associations with respect to baseline. Visuomotor-related changes in DFC with respect to baseline were estimated by using MI between the DFC and the discrete vector specifying if the trials were coming from the baseline or action condition. We used the RFX group-level approach, with p-values corrected for multiple comparisons across time and pairs of brain regions using the FDR correction. The time-averaged DFC for exhibited significant effects over the fronto-parietal network, mainly within the left hemisphere, with a strong implication of motor and premotor regions, superior parietal cortex such as strong bilateral connections within the occipital lobe 
(Figure 7A). We replicated the temporal evolution of the connectivity strength by computing the mean over pairs of brain regions exhibiting a significant contrast with the baseline (Figure 7B). In addition, the time-course averaged across pairs displayed a first peak occurring slightly after $-0.4 \mathrm{~s}$, during the movement planning phase after stimulus presentation, a dissolution of the network around os and finally the emergence of a motor-related network at $0.2 \mathrm{~s}$ when subjects performed the finger movement. In addition to the original paper, we computed the number of significant links according to the group-level model (FFX and RFX), such as approaches for correcting for multiple comparisons (FDR, Maxstat and Cluster-based across time points) (Figure 7C). Overall, the number of significant links was much larger when using the FFX model. Similarly, FDR correction led to a much larger number of significant edges, followed by the maximum statistics and finally the cluster-based correction.
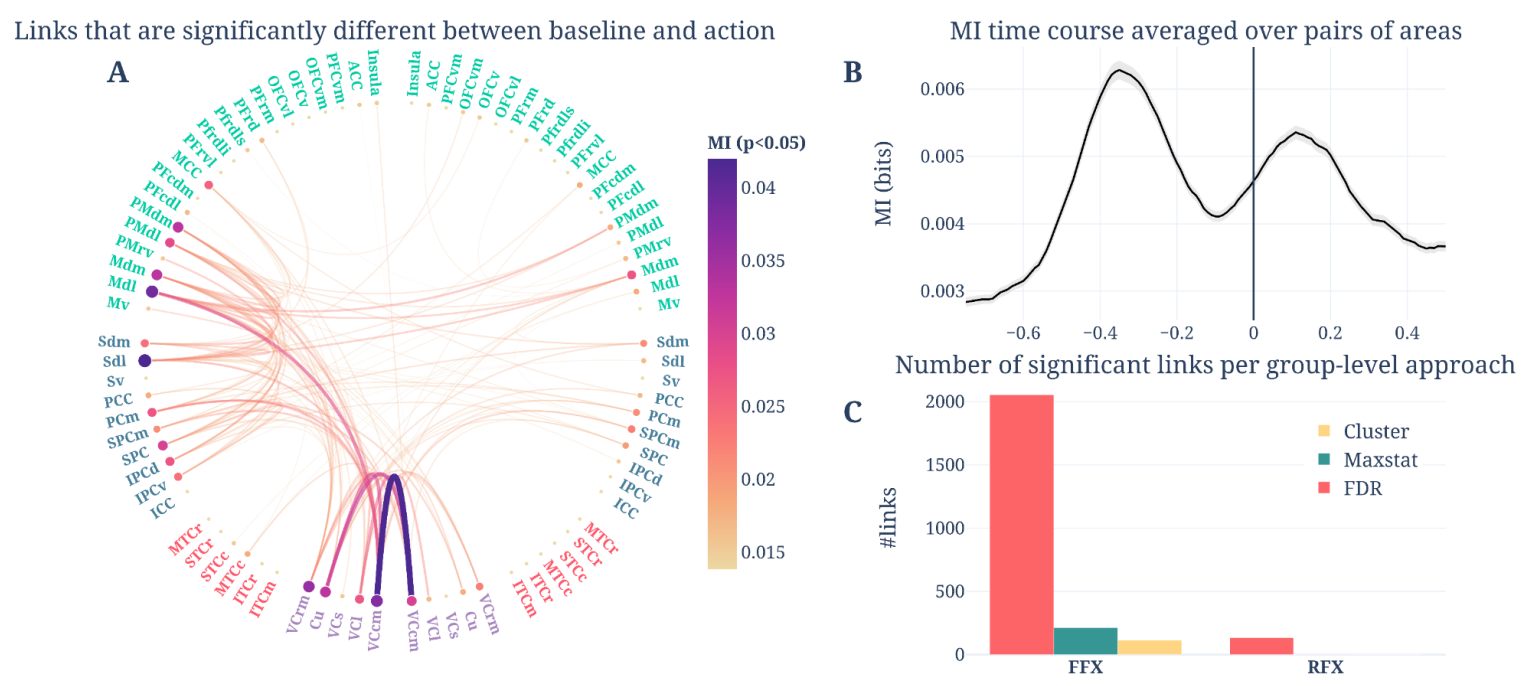

Figure 7. significant task-related undirected pairwise connectivity using $\mathrm{Ml}(A)$ circular representation of the connectivity matrix of mean Ml over time points $(B)$ time-course of mean MI and $95 \%$ confidence interval across pairs of brain regions. Data are aligned on movement onset represented here with a vertical black line at 0 seconds and the time vector represents the center of each $500 \mathrm{~ms}$ sliding window. (C) number of remaining significant links for the FFX and RFX models, respectively combined with FDR, maximum statistics and cluster-based corrections. All subplots were obtained after selecting the significant pairs of brain regions ( $p<0.05$, corrected)

Finally, we investigated task-related network properties using standard graph-theoretical metrics (Sec. 2.5.1). To this end, we estimated the single-trial network efficiency, modularity and assortativity during both action and baseline 
bioRxiv preprint doi: https://doi.org/10.1101/2021.08.14.456339; this version posted August 15, 2021. The copyright holder for this preprint (which was not certified by peer review) is the author/funder, who has granted bioRxiv a license to display the preprint in perpetuity. It is made available under aCC-BY-NC-ND 4.0 International license.

periods. As for the MI time-course of Figure 7B, all of the three metrics led to two peaks. We found a decrease of network efficiency (Figure $\mathbf{8 A}$ ) and an increase both in network modularity and assortativity (Figure 8B-C) with respect to baseline period. We then computed the $\mathrm{Ml}$ between the single-trial graph measures and a vector of labels indicating whether each trial was coming from the baseline or action periods. For the model of the group, we used a random-effect with cluster-based correction for multiple testings. For each of the three Ml time courses, we found two significant clusters prior and after movement onset reflecting significant task-related differences of efficiency, modularity and assortativity.

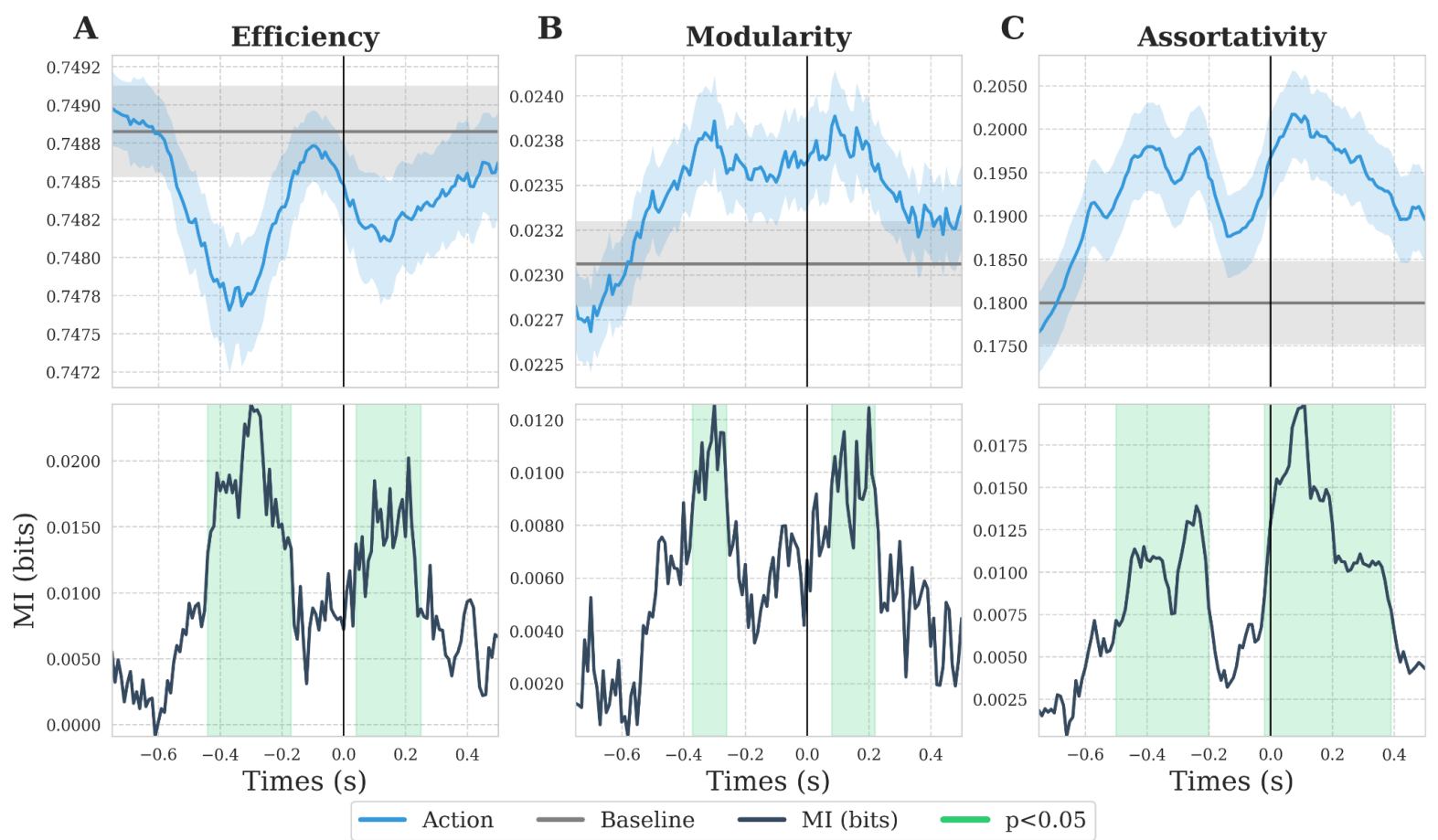

Figure 8. significant task-related graph metrics respectively measuring $(A)$ the network efficiency, $(B)$ the network modularity and (C) the network assortativity. Top row blue and gray lines represent the time courses of the graph measures respectively estimated during action and baseline. Shaded areas represent the $95 \%$ confidence interval estimated across subjects. Bottom row contains the MI time course and the significant clusters are highlighted in green (RFX $-p<0.05$ ). Data are aligned on movement onset represented here with a vertical black line at 0 .

\subsubsection{Reinforcement learning system and intracranial EEG}

We then tested our approach on intracranial HGA estimated from SEEG data recorded from the anterior Insula (aINS) in a reinforcement learning task. We computed the MI between the HGA power, estimated per bipolar contact, and the 
outcomes during both the reward $(+1 €$ vs. $+0 €)$ and punishment $(-0 €$ vs. $-1 €)$ conditions (Figure 9). As for the group-level statistics, we used a random-effect approach across contacts with cluster-based corrected p-values. Mean HGA power across subjects in the aINS showed clear modulations when receiving rewarding and punishing outcomes $(+1 €$ and $-1 €)$ in contrast to $+0 €$ and $-0 €$ outcomes (Figure 9A). Overall, the difference of HGA power between outcomes was larger during the punishment condition which was then confirmed by the magnitude of $\mathrm{Ml}$ and t-values (Figure 9B-C). The Ml showed a significant peak occurring around $500 \mathrm{~ms}$ after outcome presentation during both reward and punishment conditions. To further explore the consistency of the effect, we detected temporal clusters within-contacts and reported the proportion of contacts displaying a significant effect (Figure 9D). Interestingly, more than half of the contacts in the anterior insula showed significant differences between outcomes in the punishment condition compared to $20 \%$ during the reward.
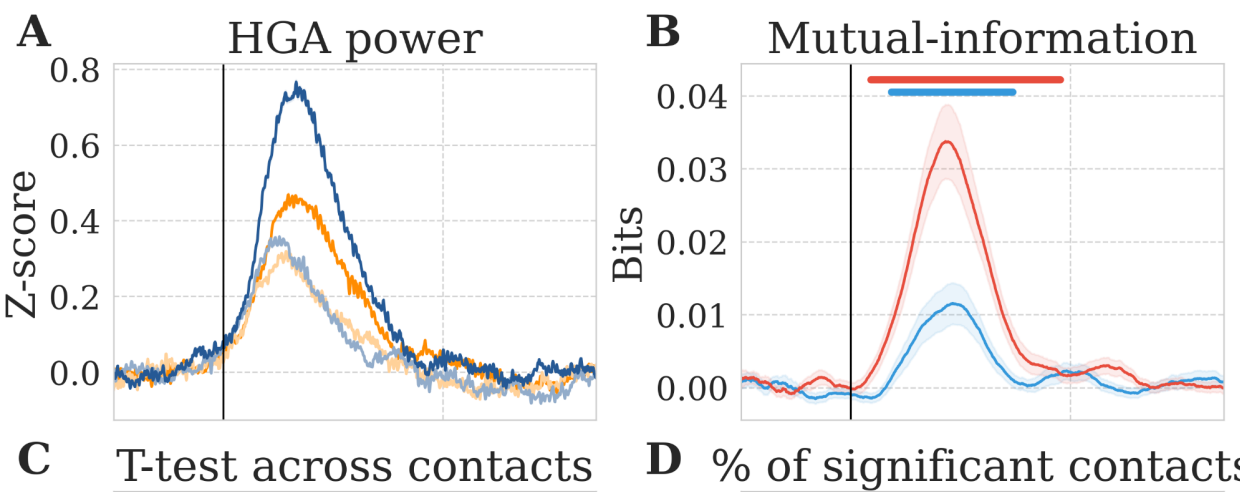

D \% of significant contacts
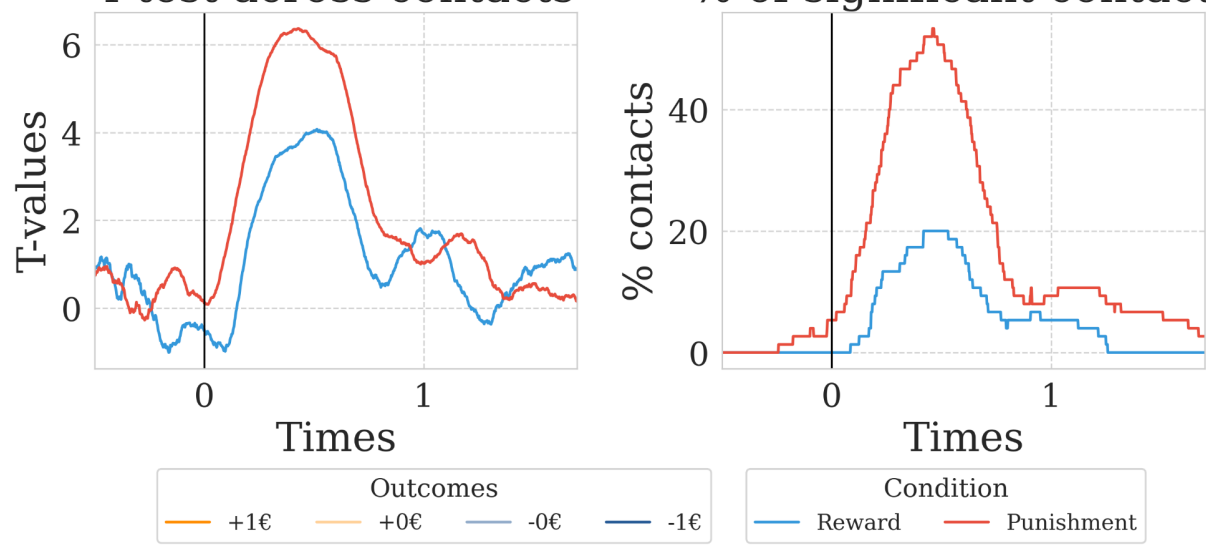

Figure 9. significant differences of HGA power between reward and punishment conditions in the anterior insula (13 subjects, 75 contacts). (A) Mean relative HGA power across subjects per outcome $(+1 €$ and $+0 €$ in dark and light orange, $-1 €$ and $-0 €$ in dark and light blue) $(B)$ Mean $\mathrm{MI}$ computed between the HGA power and the outcomes during the reward condition $\{+0 €,+1 €\}$ in blue and during the punishment condition $\{-1 €,-0 €\}$ in red. Blue and red thick lines represent the 
bioRxiv preprint doi: https://doi.org/10.1101/2021.08.14.456339; this version posted August 15,2021 . The copyright holder for this preprint (which was not certified by peer review) is the author/funder, who has granted bioRxiv a license to display the preprint in perpetuity. It is made available under aCC-BY-NC-ND 4.0 International license.

significant temporal clusters obtained, at the group-level at $p<0.05$, respectively during reward and punishment conditions. ( $C$ ), T-values obtained by computing a one-sample t-test contrasting single contact $\mathrm{Ml}$ estimations against permutation mean $(D)$ Proportion of contacts with significant effect. Vertical black line at $\mathrm{O}$ second represents the outcome presentation. 


\section{4) Discussion}

In this study, we presented a statistical framework and associated toolbox called Frites for group-level inferences using non-negative information-based measures (e.g mutual-information, decoding) to identify cognitive brain networks. Our framework extends a previous approach for group-level inferences on information-based measures (Giordano et al., 2017) to encompass neurophysiological recordings with both homogeneous spatial samplings across participants (or sessions), such as whole-brain source level data M/EEG data and spatially sparse recordings, such as intracranial sEEG, ECoG and LFPs. In addition, it allows fixed-and random-effect models to adapt to inter-subject variability and uses non-parametric permutations with test- and cluster-wise correction for $p$-values inference. We used simulated data to compare the group-level approaches and complementary methods for correcting for multiple comparisons. We then tested our approach on human data to reproduce and extend existing results based on MEG data acquired during a visuomotor task (Brovelli et al., 2017, 2015) and spatially sparse intracranial data for investigating neural correlates of probabilistic learning (Gueguen et al., 2021). Finally, we illustrated how this framework can be applied at the level of local neural activity, on pairwise functional connectivity links or on measures summarizing network properties.

\subsection{Comparison of group-level approaches}

To compare F/RFX combined with different methods for correcting for multiple testings (maximum statistics, FDR and cluster-based), we defined three simulated ground-truths covering different scenarios : (i) weak and spatially diffuse effect; (ii) strong and spatially focal effect; (iii) varying spatio-temporal effect. For assessing the acuity of the group-level approaches in retrieving the ground-truths, we used the Matthews Correlation Coefficient (MCC), which returns a single value reflecting the overall statistical performance by combining both sensitivity and specificity. Intuitively, the accuracy of both FFX and RFX increased with the number of subjects and the number of repetition per subject. For the 
same dataset (i.e., for the same number of subjects and repetitions), the FFX led to slightly higher accuracy than the RFX. However, this result should be nuanced by the fact that the first simulation used a highly reproducible effect across subjects. Indeed, when introducing inter-subject variability, FFX performance dropped until the effect started to be highly reproducible across subjects ( $80 \%$ in the context of our simulations). This is explained by the fact that FFX is a single-level model, where the effect is estimated across subjects (Penny and Holmes, 2007) and cannot adapt to the inter-subject variability. On the other hand, RFX performance was stable at every level of inter-subject variability. Indeed, the RFX is a two-level model, in which the first level explains the effect size at the single-subject level and the second fits the population-level variance. Such capabilities to adapt to the variability and being able to generalize results to the population explain why, when possible, the RFX was preferred over the FFX (Lazar et al., 2002). While the RFX approach allows generalization to new subjects and accounts for random variations from subject to subject, it also comes at the cost of a lower sensitivity compared to the FFX which should be counterbalanced by a larger cohort.

Results from simulations also showed that methods for correcting for multiple comparisons were equally impacted with increasing sample-size or by the inter-subjects variability. However, the overall performance for both maximum statistics and FDR corrections drastically decreased as the proportion of subjects having the effect was reduced. In all scenarios, the overall performance of cluster-wise correction outperformed test-wise corrections. For the cluster-based correction (Bullmore et al., 1999; Maris and Oostenveld, 2007) the test is performed over the mass of the clusters, not on their precise spatiotemporal locations, therefore the $p$-value of the cluster is not representative of the member of the cluster. As a consequence, it was recently highlighted that numerous studies are reporting overestimated precisions of the statistical claims (Sassenhagen and Draschkow, 2019). To overcome this limitation and also to overcome the definition of the cluster forming threshold, several alternatives have been proposed like the Threshold Free Cluster Enhancement (TFCE) and probabilistic TFCE (Smith and Nichols, 2009; Spisák et al., 2019) or the 
non-parametric threshold free LISA framework (Lohmann et al., 2018). Overall, TFCE has been shown to provide a better sensitivity compared to standard cluster extent (Noble et al., 2019; Pernet et al., 2015). Future work could investigate how those modern cluster definitions perform once combined to the statistical framework proposed here.

\subsection{Improving reproducibility through statistics}

It has been shown that almost every study is unique in its analytical and statistical approach (Carp, 2012). This diversity is understandable considering the variety of designs and neurophysiological recordings. However, such diversity is a potential source for the lack of reproducibility (Gilmore et al., 2017; Puoliväli et al., 2020). The diversity in statistical approaches is further extended by the eternal debate between parametric and non-parametric statistical approaches. On one side, the data-driven non-parametric approaches are more likely to provide a better control for the FWER or FDR (Hayasaka, 2003; Nichols and Hayasaka, 2003; Puoliväli et al., 2020; Thirion et al., 2007), which resulted in improved reproducibility across studies. On the other side, it was also argued that by focusing on stringent control of false positives, we might also miss many effects (Noble et al., 2019). As a consequence, imposing hard statistical constraints might be pushing the field to identifying and reporting strong localized effects while missing weak and diffuse effects (Cremers et al., 2017). Sensitivity and specificity are two intertwined statistical notions and focusing on one or the other shapes the way we see the brain. This argument goes in favor of parametric estimations, however, it was also shown that a violation of assumptions of the test can result indeed in higher but also unpredictable FWER (Eklund et al., 2016).

While there is a growing global consensus around the quality of control of non-parametric approaches, there are also classic criticisms encountered like the computational cost, the lack of reproducibility and the exchangeability hypothesis (Flandin and Friston, 2019). All three arguments are true, but should probably be nuanced. The computational cost is a technical problem that can be compensated using modern computers and programming libraries (multi-cores, multi-nodes, GPU, caching computations etc.). When living in the age where deep learning is 
applied on millions of images, it should be doable to perform 1000 up to 10000 permutations and get the results within a few minutes. Concerning the lack of reproducibility, it is true that by running the analysis several times the permutation scheme varies and therefore, some significant results might change. However, several reasons can explain this variability. First, the number of permutations is too low leading to a poor sampling of the null distribution. Then, if the result is still unstable for a larger number of permutations, it is questionable whether it is robust enough to consider being reproduced in the future. Finally, to avoid p-hacking (i.e. abusing of analysis techniques until nonsignificant results become significant (Head et al., 2015)) by changing the distribution scheme it is always possible to fix the random state of the machine, like this is done in many softwares, which leads to replicable permutations schemes (Pedregosa et al., 2011). The biggest danger of permutations probably lies in the exchangeability hypothesis (i.e., the variable we consider exchangeable in order to generate a null distribution of effects achievable by chance). For a simple contrast like baseline vs. task, where the null hypothesis is that there is no difference between the two conditions, the distribution of permutations should be constructed by permuting the labels. In other cases, choosing a permutation strategy appears sometimes as a subtle choice that requires deeper investigations like for machine-learning approaches (Combrisson and Jerbi, 2015; Valente et al., 2021). An incorrect permutation strategy (e.g., shuffling a variable that contains a structure like a temporal autocorrelation) can lead to a null distribution made of small effect size that are then going to be to easy to exceed by the true effect, leading to overconfident results (Brookshire, 2021; Liegeois et al., 2017). That being said, it is a problem the community is well aware of and much has already been written on how to choose the most appropriate permutation strategy (Maris and Oostenveld, 2007; Nichols and Holmes, 2002; Winkler et al., 2014).

Most studies have been shown to be underpowered which clearly limit our capacity to reproduce results across teams (Button et al., 2013; loannidis, 2005; Szucs and loannidis, 2017). Increasing both the number of subjects and within-subject data by data sharing is crucial for increasing the probability of detecting true effects. However, group-level models combined with 
non-parametric statistics for controlling FWER on information-based measures might also greatly contribute in improving our confidence in the reported results.

\subsection{Group-level inferences on functional connectivity estimations}

We showed that the proposed statistical framework could be used on pairwise FC to retrieve the task-related visuomotor network previously found using a linear mixed model on FC links estimated using correlation (Brovelli et al., 2017). Here, we reproduced the findings by entirely staying within the information-theoretical framework. We also found that $p$-value correction at the edge-wise level can dramatically influence the proportion of significant links according to the statistical design (i.e F/RFX and the method for correcting MC). It was shown that the choice of the parcellation scheme, with a possibly large number of links, might lead to a high proportion of false negatives (Marek et al., 2020). To improve the capacity of detecting true task-related FC, several studies brought cluster-based approaches for networks (Baggio et al., 2018; Vinokur et al., 2015; Zalesky et al., 2010) or using predefined large-scale networks (Noble and Scheinost, 2020). Those new approaches work on the same ideas that corrections for MC are going to be less stringent once informations are pooled and therefore, improve our capacity to detect true cognitive brain networks at the cost of being less precise when discussing the results (Sassenhagen and Draschkow, 2019). Those new developments should still be compatible with the statistical framework proposed here.

\subsection{Information-based as a common language for cognitive brain networks}

Measures of information are encapsulating the statistical dependency between brain data and an experimental variable. Here, for illustrating the statistical framework, we mainly used metrics from information theory by means of the Gaussian-Copula Mutual Information (GCMI) (Ince et al., 2017). GCMI has several non-negligeable advantages: (i) it can detects monotonic linear and non-linear relationships; (ii) it supports any combination of uni/multivariate of continuous/discrete variables, quantifying relationships on a common meaningful effect size scale; (iii) it contains a parametric bias-correction for unbalanced 
contrasts (i.e., when the number of data points is different); (iv) it is computationally fast. In addition, it also allows conditional MI (CMI) for removing the influence of a discrete or continuous variable. Typical use cases of CMI could be the estimation of the amount of information shared between the brain data and a behavioral variable, while conditioning of a third variable In addition, CMI is at the heart of the estimation of partial functional connectivity measures and covariance-based Granger Causality metrics. Indeed, Transfer Entropy (Schreiber, 2000), which is defined as the CMI between the past of the source and the present of the target conditioned by the past of the source, is mathematically equivalent to Granger causality for gaussian variables (Barnett et al., 2009). Overall, the GCMI is a versatile and general approach including metrics for local and network-level brain data analysis.

The framework presented here could additionally be combined with more powerful and feature-rich measures of information, such as encoding and decoding models for out-of-samples generalization, fully non-parametric kernel-based MI (Moon et al., 1995) and recent metrics from the Partial Information Framework (PID) (Ince, 2017; Wibral et al., 2017; Williams and Beer, 2010). Once combined with PID metrics, it becomes possible to investigate motivated questions like if there is redundant or synergistic information between a pair of brain regions about a variable of the task and whether this information is generalizable to the population.

Taken together, the framework presented here has the potential to investigate complex task-related modifications of local neural activity, but also more ambitious characterizations such as task-related long-range coactivations and feature-specific directed information flow (Bím et al., 2020). Linking brain networks to cognitive functions has been highlighted as one of the major challenges to overcome in the future (Bassett et al., 2020). Group-level statistics combined with rich measures of information represent one avenue to address this challenge toward the characterization of cognition. 


\subsection{Multi-scale analysis of cognitive brain networks}

We showed how the statistical framework allows population generalization from meso scale (i.e. at the single-trial activity) to the macro scale of brain-networks for characterizing both task-related functional connectivity and graph-theoretical properties. It further adapts to whole-brain parcel-based analysis of any modalities, from spatially uniform recordings like EEG and MEG to highly non-uniformly sampled invasive recordings like sEEG or ECoG. Outside of the scope shown here, the framework also has potential applications to other neurophysiological recordings like utah arrays (Brochier et al., 2018), human and animal large-scale LFPs (Dotson et al., 2018, 2017), single- and multi-unit data and neuroimaging data.

\subsection{Software implementation}

Our approach has been implemented in an open-source Python package called Frites ${ }^{6}$ (Framework for Information Theoretical analysis of Electrophysiological data and Statistics) designed for inferring cognitive brain networks by means of measures of information. The toolbox, partially developed during Brainhack events (Gau et al., 2021), includes an implementation of the proposed group-level statistical pipeline, with fixed- and random-effect models such as test- and cluster-wise corrections. By default, Frites is using a tensor-based implementation of the GCMI for faster computations. We recently added the possibility to use external functions for estimating information, like standard correlation, measures of distances, scikit-learn models (Pedregosa et al., 2011) or IDTxI non-parametric kernel-based measures of MI (Wollstadt et al., 2018). The toolbox also includes functions for estimating undirected and directed (covariance-based Granger Causality) single-trial functional connectivity. For interoperability, the main functions and classes of Frites are also supporting MNE-Python epochs-related objects. Frites is entirely written around a relatively recent package called Xarray (Hoyer and Hamman, 2017), which can be seen as a generalization of Pandas (McKinney, 2011) for labelled multidimensional arrays.

\footnotetext{
${ }^{6}$ https://brainets.aithub.io/frites/
} 
One of the features of Xarray is that it allows returning and saving outputs with attributes. Therefore, each output of Frites' function and classes also contains the inputs defined by the user such as the most relevant internal variables used for computations. The open-source accessibility of the package and the ability to track both internal and external variables, contribute to a reproducible science as it allows sharing self-contained files that include all of the parameters used for understanding and reproducing a result. From a programming perspective, we also provide online documentation ${ }^{7}$ with detailed function and input descriptions such as script and notebook examples. Code blocks are well commented and follow PEP8 guidelines for code readability. Finally, to improve long term sustainability as other recent Python packages (Combrisson et al., 2020, 2019; Meunier et al., 2020) we included both smoked (i.e. testing function's input types) and functional unit tests through a continuous integration protocol (current coverage > $84 \%$ ).

\subsection{Limitations}

The present work has several limitations and possible extensions that could be considered in future works. We used simple gaussian-based simulations to compare group-level approaches in terms of acuity of ground-truth retrieving. Even if we simulated multiple scenarios of effect distribution, it is not straightforward that the presented results will behave the same in the context of real data. The present work also investigated the number of required subjects, repetitions and proportion of subjects having the effect to achieve robust statistical inferences. Again, our simulation-based results illustrate a trend and can not be used to make sample size recommendations nor replace a proper power study (Baker et al., 2020; Maxwell et al., 2008). Future work could consider using more realistic data generation with a more sophisticated inter-subject variability modelisation. We also showed how the framework could be used on spatially non-uniform data like the sEEG. However, for both FFX and RFX inferences, measures of effect size could be unequally affected by differences in signal to noise (SNR) ratio. A possible workaround to investigate in the future could be to uniformize the SNR across brain regions by combining bootstrapping and

\footnotetext{
${ }^{7}$ https://brainets.github.io/frites/index.html
} 
stratification techniques by means of subsampling such that the distributional properties are made as similar as possible (Bosman et al., 2012).

\section{5) Conclusion}

There is a rising concern about our ability to reproduce results, partly because of underpowered studies and a wide range of analytical and statistical pipelines. Here, we proposed a statistical framework for group-level inferences on information-based measures of effect size. We demonstrated how the framework behaved according to various models of the group and corrections for multiple testing. We then illustrated the framework using information metrics coming from both the information-theory and machine-learning fields by reproducing and extending existing results on neurophysiological data. We also showed that the framework could be used at the level of brain regions, on functional connectivity and on measures of graph. The present work tends to provide more robust inferences on populations and therefore is a contributing piece toward more reproducible results.

\section{6) Acknowledgments}

EC and AB were supported by the PRC project "CausaL" (ANR-18-CE28-0016). This project/research has received funding from the European Union's Horizon 2020 Framework Programme for Research and Innovation under the Specific Grant Agreement No. 945539 (Human Brain Project SGA3). MA, AB were supported by FLAG ERA II "Joint Transnational Call 2017" - HBP - Basic and Applied Research 2, Brainsynch-Hit (ANR-17-HBPR-0001). RB acknowledges support through a PhD Scholarship awarded by the Neuroschool. This work has received support from the French government under the Programme Investissements d'Avenir, Initiative d'Excellence d'Aix-Marseille Université via A*Midex (AMX-19-IET-004) and ANR (ANR-17-EURE-0029) funding. RAAI was supported by the Wellcome Trust [214120/Z/18/Z]. JB was supported by ANR-17-CE37-0018 and ANR-18-CE28-0016. Centre de Calcul Intensif 
bioRxiv preprint doi: https://doi.org/10.1101/2021.08.14.456339; this version posted August 15,2021 . The copyright holder for this preprint

(which was not certified by peer review) is the author/funder, who has granted bioRxiv a license to display the preprint in perpetuity. It is made available under aCC-BY-NC-ND 4.0 International license.

d'Aix-Marseille is acknowledged for granting access to its high performance computing resources. 


\section{References}

Allefeld, C., Görgen, K., Haynes, J.-D., 2016. Valid population inference for information-based imaging: From the second-level t-test to prevalence inference. Neurolmage 141, 378-392. https://doi.org/10.1016/j.neuroimage.2016.07.040

Arnold, B., Bowler, L., Gibson, S., Herterich, P., Higman, R., Krystalli, A., Morley, A., O’Reilly, M., Whitaker, K., others, 2019. The turing Way: a handbook for reproducible data science. Zenodo.

Auzias, G., Coulon, O., Brovelli, A., 2016. MarsAtlas : A cortical parcellation atlas for functional mapping: MarsAtlas. Hum. Brain Mapp. 37, 1573-1592. https://doi.org/10.1002/hbm.23121

Baggio, H.C., Abos, A., Segura, B., Campabadal, A., Garcia-Diaz, A., Uribe, C., Compta, Y., Marti, M.J., Valldeoriola, F., Junque, C., 2018. Statistical inference in brain graphs using threshold-free network-based statistics. Wiley Online Library.

Baker, D.H., Vilidaite, G., Lygo, F.A., Smith, A.K., Flack, T.R., Gouws, A.D., Andrews, T.J., 2020. Power contours: Optimising sample size and precision in experimental psychology and human neuroscience. Psychol. Methods.

Barnett, L., Barrett, A.B., Seth, A.K., 2009. Granger causality and transfer entropy are equivalent for Gaussian variables. Phys. Rev. Lett. 103, 238701.

Bassett, D.S., Cullen, K.E., Eickhoff, S.B., Farah, M.J., Goda, Y., Haggard, P., Hu, H., Hurd, Y.L., Josselyn, S.A., Khakh, B.S., Knoblich, J.A., Poirazi, P., Poldrack, R.A., Prinz, M., Roelfsema, P.R., Spires-Jones, T.L., Sur, M., Ueda, H.R., 2020. Reflections on the past two decades of neuroscience. Nat. Rev. Neurosci. 21, 524-534. https://doi.org/10.1038/s41583-020-0363-6

Bastin, J., Deman, P., David, O., Gueguen, M., Benis, D., Minotti, L., Hoffman, D., Combrisson, E., Kujala, J., Perrone-Bertolotti, M., 2016. Direct Recordings from Human Anterior Insula Reveal its Leading Role within the Error-Monitoring Network. Cereb. Cortex 1, 13.

Battaglia, D., Brovelli, A., 2020. Functional connectivity and neuronal dynamics: insights from computational methods. Cogn. Neurosci.

Beirlant, J., Dudewicz, E.J., Györfi, L., Van der Meulen, E.C., 1997. Nonparametric entropy estimation: An overview. Int. J. Math. Stat. Sci. 6, 17-39.

Besserve, M., Lowe, S.C., Logothetis, N.K., Schölkopf, B., Panzeri, S., 2015. Shifts of gamma phase across primary visual cortical sites reflect dynamic stimulus-modulated information transfer. PLoS Biol 13, e1002257.

Bijsterbosch, J., Harrison, S.J., Jbabdi, S., Woolrich, M., Beckmann, C., Smith, S., Duff, E.P., 2020. Challenges and future directions for representations of functional brain organization. Nat. Neurosci. 23, 1484-1495. https://doi.org/10.1038/s41593-020-00726-z

Bím, J., De Feo, V., Chicharro, D., Bieler, M., Hanganu-Opatz, I.L., Brovelli, A., Panzeri, S., 2020. A Non-negative Measure Of Feature-Related Information Transfer Between Neural Signals. bioRxiv 758128.

Borst, A., Theunissen, F.E., 1999. Information theory and neural coding. Nat. Neurosci. 2, 947-957.

Bosman, C.A., Schoffelen, J.-M., Brunet, N., Oostenveld, R., Bastos, A.M., Womelsdorf, T., Rubehn, B., Stieglitz, T., De Weerd, P., Fries, P., 2012. Attentional stimulus selection through selective synchronization between monkey visual areas. Neuron 75, 875-888.

Bressler, S.L., Menon, V., 2010. Large-scale brain networks in cognition: emerging methods and principles. Trends Cogn. Sci. 14, 277-290. https://doi.org/10.1016/j.tics.2010.04.004

Bressler, S.L., Seth, A.K., 2011. Wiener-Granger causality: a well established methodology. Neuroimage 58, 323-329.

Brochier, T., Zehl, L., Hao, Y., Duret, M., Sprenger, J., Denker, M., Grün, S., Riehle, A., 2018. Massively parallel recordings in macaque motor cortex during an instructed delayed reach-to-grasp task. Sci. Data 5, 1-23.

Brookshire, G., 2021. Re-evaluating rhythmic attentional switching: Spurious oscillations from shuffling-in-time. BioRxiv.

Brovelli, A., Badier, J.-M., Bonini, F., Bartolomei, F., Coulon, O., Auzias, G., 2017. Dynamic Reconfiguration of Visuomotor-Related Functional Connectivity Networks. J. Neurosci. 37, 
839-853. https://doi.org/10.1523/JNEUROSCI.1672-16.2016

Brovelli, A., Ding, M., Ledberg, A., Chen, Y., Nakamura, R., Bressler, S.L., 2004. Beta oscillations in a large-scale sensorimotor cortical network: Directional influences revealed by Granger causality. Proc. Natl. Acad. Sci. 101, 9849-9854. https://doi.org/10.1073/pnas.0308538101

Brovelli, X.A., Chicharro, D., Badier, J.-M., Wang, H., Jirsa, V., 2015. Characterization of Cortical Networks and Corticocortical Functional Connectivity Mediating Arbitrary Visuomotor Mapping. J. Neurosci. 35, 12643-12658.

Bullmore, E.T., Suckling, J., Overmeyer, S., Rabe-Hesketh, S., Taylor, E., Brammer, M.J., 1999. Global, voxel, and cluster tests, by theory and permutation, for a difference between two groups of structural MR images of the brain. IEEE Trans. Med. Imaging 18, 32-42. https://doi.org/10.1109/42.750253

Button, K.S., Ioannidis, J.P., Mokrysz, C., Nosek, B.A., Flint, J., Robinson, E.S., Munafò, M.R., 2013. Power failure: why small sample size undermines the reliability of neuroscience. Nat. Rev. Neurosci. 14, 365-376.

Cao, J., Zhang, S., 2014. Multiple Comparison Procedures. JAMA 312, 543-544. https://doi.org/10.1001/jama.2014.9440

Carp, J., 2012. The secret lives of experiments: methods reporting in the fMRI literature. Neuroimage 63, 289-300.

Chicco, D., Jurman, G., 2020. The advantages of the Matthews correlation coefficient (MCC) over F1 score and accuracy in binary classification evaluation. BMC Genomics 21, 6. https://doi.org/10.1186/s12864-019-6413-7

Cogan, G.B., Poeppel, D., Cogan, G.B., 2011. A mutual information analysis of neural coding of speech by low 4 frequency MEG phase information 5 .

Combrisson, E., Jerbi, K., 2015. Exceeding chance level by chance: The caveat of theoretical chance levels in brain signal classification and statistical assessment of decoding accuracy. J. Neurosci. Methods 250, 126-136. https://doi.org/10.1016/j.jneumeth.2015.01.010

Combrisson, E., Nest, T., Brovelli, A., Ince, R.A.A., Soto, J.L.P., Guillot, A., Jerbi, K., 2020. Tensorpac: An open-source Python toolbox for tensor-based phase-amplitude coupling measurement in electrophysiological brain signals. PLoS Comput. Biol. 16, e1008302. https://doi.org/10.1371/journal.pcbi.1008302

Combrisson, E., Perrone-Bertolotti, M., Soto, J.L., Alamian, G., Kahane, P., Lachaux, J.-P., Guillot, A., Jerbi, K., 2017. From intentions to actions: Neural oscillations encode motor processes through phase, amplitude and phase-amplitude coupling. Neurolmage 147, 473-487. https://doi.org/10.1016/j.neuroimage.2016.11.042

Combrisson, E., Vallat, R., O'Reilly, C., Jas, M., Pascarella, A., Saive, A., Thiery, T., Meunier, D., Altukhov, D., Lajnef, T., Ruby, P., Guillot, A., Jerbi, K., 2019. Visbrain: A Multi-Purpose GPU-Accelerated Open-Source Suite for Multimodal Brain Data Visualization. Front. Neuroinformatics 13. https://doi.org/10.3389/fninf.2019.00014

Cover, T.M., Thomas, J.A., 1991. Elements of information theory. New York: Wiley.

Cremers, H.R., Wager, T.D., Yarkoni, T., 2017. The relation between statistical power and inference in fMRI. PLOS ONE 12, e0184923. https://doi.org/10.1371/journal.pone.0184923

Ding, M., Chen, Y., Bressler, S., 2006. Granger causality: Basic theory and application to neuroscience. 2006. Handb. Time Ser. Anal. Internet Wiley Wienheim.

Dotson, N.M., Hoffman, S.J., Goodell, B., Gray, C.M., 2018. Feature-based visual short-term memory is widely distributed and hierarchically organized. Neuron 99, 215-226.

Dotson, N.M., Hoffman, S.J., Goodell, B., Gray, C.M., 2017. A large-scale semi-chronic microdrive recording system for non-human primates. Neuron 96, 769-782.

Eklund, A., Nichols, T.E., Knutsson, H., 2016. Cluster failure: Why fMRI inferences for spatial extent have inflated false-positive rates. Proc. Natl. Acad. Sci. 113, 7900-7905. https://doi.org/10.1073/pnas.1602413113

Flandin, G., Friston, K.J., 2019. Analysis of family-wise error rates in statistical parametric mapping using random field theory. Hum. Brain Mapp. 40, 2052-2054.

Friston, K.J., Holmes, A.P., Worsley, K.J., Poline, J.-P., Frith, C.D., Frackowiak, R.S., 1994. Statistical parametric maps in functional imaging: a general linear approach. Hum. Brain Mapp. 2, 189-210.

Gau, R., Noble, S., Hever, K., Bottenhorn, K.L., Bilgin, I.P., Yang, Y.-F., Huntenburg, J.M., Bayer, J.M., Bethlehem, R.A., Rhoads, S.A., others, 2021. Brainhack: Developing a culture of open, inclusive, community-driven neuroscience. Neuron 109, 1769-1775. 
Geman, S., Bienenstock, E., Doursat, R., 1992. Neural networks and the bias/variance dilemma. Neural Comput. 4, 1-58.

Genovese, C.R., Lazar, N.A., Nichols, T., 2002. Thresholding of statistical maps in functional neuroimaging using the false discovery rate. Neuroimage 870-878.

Gilmore, R.O., Diaz, M.T., Wyble, B.A., Yarkoni, T., 2017. Progress toward openness, transparency, and reproducibility in cognitive neuroscience. Ann. N. Y. Acad. Sci. 1396, 5-18.

Giordano, B.L., Ince, R.A., Gross, J., Schyns, P.G., Panzeri, S., Kayser, C., 2017. Contributions of local speech encoding and functional connectivity to audio-visual speech perception. Elife 6, e24763.

Glaser, J.I., Benjamin, A.S., Farhoodi, R., Kording, K.P., 2019. The roles of supervised machine learning in systems neuroscience. Prog. Neurobiol. 175, 126-137. https://doi.org/10.1016/j.pneurobio.2019.01.008

Gramfort, A., Luessi, M., Larson, E., Engemann, D.A., Strohmeier, D., Brodbeck, C., Goj, R., Jas, M., Brooks, T., Parkkonen, L., others, 2013. MEG and EEG data analysis with MNE-Python. Front. Neurosci. 7, 267.

Gross, J., Hoogenboom, N., Thut, G., Schyns, P., Panzeri, S., Belin, P., Garrod, S., 2013. Speech rhythms and multiplexed oscillatory sensory coding in the human brain. PLoS Biol. 11, e1001752.

Gueguen, M.C., Lopez-Persem, A., Billeke, P., Lachaux, J.-P., Rheims, S., Kahane, P., Minotti, L., David, O., Pessiglione, M., Bastin, J., 2021. Anatomical dissociation of intracerebral signals for reward and punishment prediction errors in humans. Nat. Commun. 12, 1-12.

Hayasaka, S., 2003. Validating cluster size inference: random field and permutation methods. Neurolmage 20, 2343-2356. https://doi.org/10.1016/j.neuroimage.2003.08.003

Head, M.L., Holman, L., Lanfear, R., Kahn, A.T., Jennions, M.D., 2015. The extent and consequences of p-hacking in science. PLoS Biol. 13, e1002106.

Holdgraf, C., Appelhoff, S., Bickel, S., Bouchard, K., D’Ambrosio, S., David, O., Devinsky, O., Dichter, B., Flinker, A., Foster, B.L., others, 2019. iEEG-BIDS, extending the Brain Imaging Data Structure specification to human intracranial electrophysiology. Sci. Data 6, 1-6.

Holmes, A.P., Blair, R., Watson, J., Ford, I., 1996. Nonparametric analysis of statistic images from functional mapping experiments. J. Cereb. Blood Flow Metab. 16, 7-22.

Hoyer, S., Hamman, J., 2017. xarray: ND labeled arrays and datasets in Python. J. Open Res. Softw. 5.

Ince, R.A., 2017. Measuring multivariate redundant information with pointwise common change in surprisal. Entropy 19, 318.

Ince, R.A., Jaworska, K., Gross, J., Panzeri, S., Van Rijsbergen, N.J., Rousselet, G.A., Schyns, P.G., 2016. The deceptively simple N170 reflects network information processing mechanisms involving visual feature coding and transfer across hemispheres. Cereb. Cortex 26, 4123-4135.

Ince, R.A., Van Rijsbergen, N.J., Thut, G., Rousselet, G.A., Gross, J., Panzeri, S., Schyns, P.G., 2015. Tracing the flow of perceptual features in an algorithmic brain network. Sci. Rep. 5, 1-17.

Ince, R.A.A., Giordano, B.L., Kayser, C., Rousselet, G.A., Gross, J., Schyns, P.G., 2017. A statistical framework for neuroimaging data analysis based on mutual information estimated via a gaussian copula: Gaussian Copula Mutual Information. Hum. Brain Mapp. 38, 1541-1573. https://doi.org/10.1002/hbm.23471

Ince, R.A.A., Mazzoni, A., Bartels, A., Logothetis, N.K., Panzeri, S., 2012. A novel test to determine the significance of neural selectivity to single and multiple potentially correlated stimulus features. J. Neurosci. Methods 210, 49-65. https://doi.org/10.1016/j.jneumeth.2011.11.013

loannidis, J.P., 2005. Why most published research findings are false. PLoS Med. 2, e124.

Ioannidis, J.P., Munafo, M.R., Fusar-Poli, P., Nosek, B.A., David, S.P., 2014. Publication and other reporting biases in cognitive sciences: detection, prevalence, and prevention. Trends Cogn. Sci. 18, 235-241.

Jeong, J., Gore, J.C., Peterson, B.S., 2001. Mutual information analysis of the EEG in patients with Alzheimer's disease. Clin. Neurophysiol. 112, 827-835. https://doi.org/10.1016/S1388-2457(01)00513-2

Jerbi, K., Combrisson, E., Dalal, S.S., Vidal, J.R., Hamame, C.M., Bertrand, O., Berthoz, A., Kahane, P., Lachaux, J.-P., others, 2013. Decoding cognitive states and motor intentions from intracranial EEG: How promising is high-frequency brain activity for brain-machine interfaces? Epilepsy Behav. 28, 283-302. 
Jerbi, K., Ossandon, T., Hamamé, C.M., Senova, S., Dalal, S.S., Jung, J., Minotti, L., Bertrand, O., Berthoz, A., Kahane, P., Lachaux, J.-P., 2009. Task-related gamma-band dynamics from an intracerebral perspective: Review and implications for surface EEG and MEG. Hum. Brain Mapp. 30, 1758-1771. https://doi.org/10.1002/hbm.20750

Kriegeskorte, N., Bandettini, P., 2007. Analyzing for information, not activation, to exploit high-resolution fMRI. Neurolmage 38, 649-662.

Kriegeskorte, N., Goebel, R., Bandettini, P., 2006. Information-based functional brain mapping. Proc. Natl. Acad. Sci. 103, 3863-3868. https://doi.org/10.1073/pnas.0600244103

Lazar, N.A., Luna, B., Sweeney, J.A., Eddy, W.F., 2002. Combining brains: a survey of methods for statistical pooling of information. Neuroimage 16, 538-550.

Liegeois, R., Laumann, T.O., Snyder, A.Z., Zhou, J., Yeo, B.T., 2017. Interpreting temporal fluctuations in resting-state functional connectivity MRI. Neuroimage.

Lindquist, M.A., Mejia, A., 2015. Zen and the art of multiple comparisons. Psychosom. Med. 77, 114.

Lohmann, G., Stelzer, J., Lacosse, E., Kumar, V.J., Mueller, K., Kuehn, E., Grodd, W., Scheffler, K., 2018. LISA improves statistical analysis for fMRI. Nat. Commun. 9, 1-9.

Magri, C., Whittingstall, K., Singh, V., Logothetis, N.K., Panzeri, S., 2009. A toolbox for the fast information analysis of multiple-site LFP, EEG and spike train recordings. BMC Neurosci. 10, 81.

Marek, S., Tervo-Clemmens, B., Calabro, F.J., Montez, D.F., Kay, B.P., Hatoum, A.S., Donohue, M.R., Foran, W., Miller, R.L., Feczko, E., Miranda-Dominguez, O., Graham, A.M., Earl, E.A., Perrone, A.J., Cordova, M., Doyle, O., Moore, L.A., Conan, G., Uriarte, J., Snider, K., Tam, A., Chen, J., Newbold, D.J., Zheng, A., Seider, N.A., Van, A.N., Laumann, T.O., Thompson, W.K., Greene, D.J., Petersen, S.E., Nichols, T.E., Yeo, B.T.T., Barch, D.M., Garavan, H., Luna, B., Fair, D.A., Dosenbach, N.U.F., 2020. Towards Reproducible Brain-Wide Association Studies (preprint). Neuroscience. https://doi.org/10.1101/2020.08.21.257758

Maris, E., Oostenveld, R., 2007. Nonparametric statistical testing of EEG- and MEG-data. J. Neurosci. Methods 164, 177-190. https://doi.org/10.1016/j.jneumeth.2007.03.024

Massey, J., 1990. Causality, feedback and directed information, in: Proc. Int. Symp. Inf. Theory Applic.(ISITA-90). Citeseer, pp. 303-305.

Matthews, B.W., 1975. Comparison of the predicted and observed secondary structure of T4 phage lysozyme. Biochim. Biophys. Acta BBA-Protein Struct. 405, 442-451.

Maxwell, S.E., Kelley, K., Rausch, J.R., 2008. Sample size planning for statistical power and accuracy in parameter estimation. Annu Rev Psychol 59, 537-563.

McKinney, W., 2011. pandas: a foundational Python library for data analysis and statistics. Python High Perform. Sci. Comput. 1-9.

Mensen, A., Khatami, R., 2013. Advanced EEG analysis using threshold-free cluster-enhancement and non-parametric statistics. Neurolmage 67, 111-118. https://doi.org/10.1016/j.neuroimage.2012.10.027

Meunier, D., Pascarella, A., Altukhov, D., Jas, M., Combrisson, E., Lajnef, T., Bertrand-Dubois, D., Hadid, V., Alamian, G., Alves, J., others, 2020. NeuroPycon: An open-source Python toolbox for fast multi-modal and reproducible brain connectivity pipelines. Neurolmage 117020.

Moon, Y.-I., Rajagopalan, B., Lall, U., 1995. Estimation of mutual information using kernel density estimators. Phys. Rev. E 52, 2318.

Mumford, J.A., Nichols, T., 2006. Modeling and inference of multisubject fMRI data. IEEE Eng. Med. Biol. Mag. 25, 42-51. https://doi.org/10.1109/MEMB.2006.1607668

Nichols, T., Hayasaka, S., 2003. Controlling the familywise error rate in functional neuroimaging: a comparative review. Stat. Methods Med. Res. 12, 419-446. https://doi.org/10.1191/0962280203sm341ra

Nichols, T.E., Holmes, A.P., 2002. Nonparametric permutation tests for functional neuroimaging: a primer with examples. Hum. Brain Mapp. 15, 1-25.

Niso, G., Gorgolewski, K.J., Bock, E., Brooks, T.L., Flandin, G., Gramfort, A., Henson, R.N., Jas, M., Litvak, V., Moreau, J.T., Oostenveld, R., Schoffelen, J.-M., Tadel, F., Wexler, J., Baillet, S., 2018. MEG-BIDS, the brain imaging data structure extended to magnetoencephalography [WWW Document]. Sci. Data. https://doi.org/10.1038/sdata.2018.110

Noble, S., Scheinost, D., 2020. The constrained network-based statistic: a new level of inference for neuroimaging, in: International Conference on Medical Image Computing and Computer-Assisted Intervention. Springer, pp. 458-468. 
Noble, S., Scheinost, D., Constable, R.T., 2019. Cluster failure or power failure? Evaluating sensitivity in cluster-level inference. Neurolmage 116468. https://doi.org/10.1016/j.neuroimage.2019.116468

Ostwald, D., Bagshaw, A.P., 2011. Information theoretic approaches to functional neuroimaging. Magn. Reson. Imaging 29, 1417-1428. https://doi.org/10.1016/j.mri.2011.07.013

Palminteri, S., Justo, D., Jauffret, C., Pavlicek, B., Dauta, A., Delmaire, C., Czernecki, V., Karachi, C., Capelle, L., Durr, A., Pessiglione, M., 2012. Critical Roles for Anterior Insula and Dorsal Striatum in Punishment-Based Avoidance Learning. Neuron 76, 998-1009. https://doi.org/10.1016/j.neuron.2012.10.017

Panzeri, S., Harvey, C.D., Piasini, E., Latham, P.E., Fellin, T., 2017. Cracking the Neural Code for Sensory Perception by Combining Statistics, Intervention, and Behavior. Neuron 93, 491-507. https://doi.org/10.1016/j.neuron.2016.12.036

Panzeri, S., Magri, C., Logothetis, N.K., 2008. On the use of information theory for the analysis of the relationship between neural and imaging signals. Magn. Reson. Imaging 26, 1015-1025. https://doi.org/10.1016/j.mri.2008.02.019

Pavlov, Y.G., Adamian, N., Appelhoff, S., Arvaneh, M., Benwell, C.S., Beste, C., Bland, A.R., Bradford, D.E., Bublatzky, F., Busch, N.A., others, 2021. \# EEGManyLabs: Investigating the replicability of influential EEG experiments. Cortex.

Pedregosa, F., Varoquaux, G., Gramfort, A., Michel, V., Thirion, B., Grisel, O., Blondel, M., Prettenhofer, P., Weiss, R., Dubourg, V., Vanderplas, J., Passos, A., Cournapeau, D., Brucher, M., Perrot, M., Duchesnay, E., 2011. Scikit learn : Machine Learning in Python. J. Mach. Learn. Res. 12, 2825-2830.

Penny, W., Holmes, A., 2007. Random effects analysis. Stat. Parametr. Mapp. Anal. Funct. Brain Images 156, 165.

Pernet, C., Garrido, M., Gramfort, A., Maurits, N., Michel, C., Pang, E., Salmelin, R., Schoffelen, J.M., Valdes-Sosa, P.A., Puce, A., 2018. Best practices in data analysis and sharing in neuroimaging using MEEG.

Pernet, C., Garrido, M.I., Gramfort, A., Maurits, N., Michel, C.M., Pang, E., Salmelin, R., Schoffelen, J.M., Valdes-Sosa, P.A., Puce, A., 2020. Issues and recommendations from the OHBM COBIDAS MEEG committee for reproducible EEG and MEG research. Nat. Neurosci. 23, $1473-1483$.

Pernet, C.R., Appelhoff, S., Gorgolewski, K.J., Flandin, G., Phillips, C., Delorme, A., Oostenveld, R., 2019. EEG-BIDS, an extension to the brain imaging data structure for electroencephalography. Sci. Data 6, 1-5.

Pernet, C.R., Latinus, M., Nichols, T.E., Rousselet, G.A., 2015. Cluster-based computational methods for mass univariate analyses of event-related brain potentials/fields: A simulation study. J. Neurosci. Methods 250, 85-93. https://doi.org/10.1016/j.jneumeth.2014.08.003

Pessiglione, M., Seymour, B., Flandin, G., Dolan, R.J., Frith, C.D., 2006. Dopamine-dependent prediction errors underpin reward-seeking behaviour in humans. Nature 442, 1042-1045. https://doi.org/10.1038/nature05051

Poldrack, R.A., Baker, C.I., Durnez, J., Gorgolewski, K.J., Matthews, P.M., Munafò, M.R., Nichols, T.E., Poline, J.-B., Vul, E., Yarkoni, T., 2017. Scanning the horizon: towards transparent and reproducible neuroimaging research. Nat. Rev. Neurosci. 18, 115-126. https://doi.org/10.1038/nrn.2016.167

Poldrack, R.A., Whitaker, K., Kennedy, D., 2020. Introduction to the special issue on reproducibility in neuroimaging. Neurolmage 218, 116357. https://doi.org/10.1016/j.neuroimage.2019.116357

Puoliväli, T., Palva, S., Palva, J.M., 2020. Influence of multiple hypothesis testing on reproducibility in neuroimaging research: A simulation study and Python-based software. J. Neurosci. Methods 337, 108654. https://doi.org/10.1016/j.jneumeth.2020.108654

Quiroga, R.Q., Panzeri, S., 2009. Extracting information from neuronal populations: information theory and decoding approaches. Nat. Rev. Neurosci. 10, 173.

Reid, A.T., 2019. Advancing functional connectivity research from association to causation. Nat. Neurosci. 22, 10.

Roels, S.P., Loeys, T., Moerkerke, B., 2016. Evaluation of Second-Level Inference in fMRI Analysis. Comput. Intell. Neurosci. 2016, 1-22. https://doi.org/10.1155/2016/1068434

Rubinov, M., Sporns, O., 2010. Complex network measures of brain connectivity: uses and interpretations. Neuroimage 52, 1059-1069. 
Sassenhagen, J., Draschkow, D., 2019. Cluster-based permutation tests of MEG/EEG data do not establish significance of effect latency or location. Psychophysiology e13335. https://doi.org/10.1111/psyp.13335

Schreiber, T., 2000. Measuring information transfer. Phys. Rev. Lett. 85, 461.

Schyns, P.G., Thut, G., Gross, J., 2011. Cracking the code of oscillatory activity. PLoS Biol. 9, e1001064.

Seth, A.K., Barrett, A.B., Barnett, L., 2015. Granger causality analysis in neuroscience and neuroimaging. J. Neurosci. 35, 3293-3297.

Shapiro, D.E., 1999. The interpretation of diagnostic tests. Stat. Methods Med. Res. 8, 113-134. https://doi.org/10.1177/096228029900800203

Smith, S., Nichols, T., 2009. Threshold-free cluster enhancement: Addressing problems of smoothing, threshold dependence and localisation in cluster inference. Neurolmage 44, 83-98. https://doi.org/10.1016/j.neuroimage.2008.03.061

Spisák, T., Spisák, Z., Zunhammer, M., Bingel, U., Smith, S., Nichols, T., Kincses, T., 2019. Probabilistic TFCE: A generalized combination of cluster size and voxel intensity to increase statistical power. Neurolmage 185, 12-26.

Szucs, D., loannidis, J.P., 2017. Empirical assessment of published effect sizes and power in the recent cognitive neuroscience and psychology literature. PLoS Biol. 15, e2000797.

Thirion, B., Pinel, P., Mériaux, S., Roche, A., Dehaene, S., Poline, J.-B., 2007. Analysis of a large fMRI cohort: Statistical and methodological issues for group analyses. Neurolmage 35, 105-120. https://doi.org/10.1016/j.neuroimage.2006.11.054

Timme, N.M., Lapish, C., 2018. A Tutorial for Information Theory in Neuroscience. eNeuro 5. https://doi.org/10.1523/ENEURO.0052-18.2018

Treves, A., Panzeri, S., 1995. The upward bias in measures of information derived from limited data samples. Neural Comput. 7, 399-407.

Valente, G., Castellanos, A.L., Hausfeld, L., De Martino, F., Formisano, E., 2021. Cross-validation and permutations in MVPA: validity of permutation strategies and power of cross-validation schemes. Neurolmage 118145.

Varela, F., Lachaux, J.-P., Rodriguez, E., Martinerie, J., 2001. The brainweb: Phase synchronization and large-scale integration. Nat. Rev. Neurosci. 2, 229-239. https://doi.org/10.1038/35067550

Vicente, R., Wibral, M., Lindner, M., Pipa, G., 2011. Transfer entropy-a model-free measure of effective connectivity for the neurosciences. J. Comput. Neurosci. 30, 45-67.

Vihinen, M., 2012. How to evaluate performance of prediction methods? Measures and their interpretation in variation effect analysis, in: BMC Genomics. BioMed Central, pp. 1-10.

Vinokur, L., Zalesky, A., Raffelt, D., Smith, R., Connelly, A., 2015. A Novel Threshold-Free Network-Based Statistics Method: Demonstration using Simulated Pathology. Organ. Hum. Brain Mapp. 4144.

von der Malsburg, C.E., Phillps, W.A., Singer, W.E., 2010. Dynamic coordination in the brain: From neurons to mind. MIT Press.

Wibral, M., Lizier, J.T., Priesemann, V., 2015. Bits from Brains for Biologically Inspired Computing. Front. Robot. Al 2. https://doi.org/10.3389/frobt.2015.00005

Wibral, M., Priesemann, V., Kay, J.W., Lizier, J.T., Phillips, W.A., 2017. Partial information decomposition as a unified approach to the specification of neural goal functions. Brain Cogn. 112, 25-38.

Williams, P.L., Beer, R.D., 2010. Nonnegative Decomposition of Multivariate Information. ArXiv10042515 Math-Ph Physicsphysics Q-Bio.

Winkler, A.M., Ridgway, G.R., Webster, M.A., Smith, S.M., Nichols, T.E., 2014. Permutation inference for the general linear model. Neuroimage 92, 381-397.

Wollstadt, P., Lizier, J.T., Vicente, R., Finn, C., Martinez-Zarzuela, M., Mediano, P., Novelli, L., Wibral, M., 2018. IDTxl: The Information Dynamics Toolkit xl: a Python package for the efficient analysis of multivariate information dynamics in networks. ArXiv Prepr. ArXiv180710459.

Zalesky, A., Fornito, A., Bullmore, E.T., 2010. Network-based statistic: identifying differences in brain networks. Neuroimage 53, 1197-1207. 